\title{
INDEX OF SURFACE-WATER STATIONS IN TEXAS, JANUARY 1986
}

Compiled by E.R. Carrillo, H.D. Buckner, and Jack Rawson

U.S. GEOLOGICAL SURVEY

Open-File Report 86-235

Austin, Texas

1986 


\section{CONTENTS}

Page

Introduction $\ldots \ldots \ldots \ldots \ldots \ldots \ldots \ldots \ldots \ldots \ldots \ldots \ldots \ldots \ldots \ldots \ldots \ldots \ldots \ldots \ldots \ldots$

Description of surface-water stations.......................

Availability of data................................. 4

\section{ILLUSTRATIONS}

Plate 1. Map showing the locations of active surface-water stations in Texas, January $1986 . \ldots \ldots \ldots \ldots \ldots \ldots \ldots \ldots \ldots$ In pocket

2. Map showing the locations of active partial-record surfacewater stations in Texas, January 1986............... In pocket

TABLES

Table 1. Streamflow, quality, reservoir-content, and partial-record stations maintained by the U.S. Geological Survey in cooperation with State and Federal agencies............ 
INDEX OF SURFACE-WATER STATIONS IN TEXAS

JANUARY 1986

Compiled by

E. R. Carrillo, H. D. Buckner, and Jack Rawson

\section{INTRODUCTION}

The U.S. Geological Survey's investigations of the water resources of Texas are conducted in cooperation with the Texas Department of Water Resources, river authorities, cities, counties, U.S. Army Corps of Engineers, U.S. Bureau of Reclamation, International Boundary and Water Commission, and others.

Investigations are under the general direction of $C$. W. Boning, District Chief, Texas District. The Texas District office is located in the Federal Building, 300 East 8th Street, Austin, Texas 78701.

Information regarding hydrologic data c0llected within their respective areas may be obtained from the subdistrict offices listed below:

W. E. Reeves, Chief

Field Headquarters

U.S. Geological Survey, WRD

300 E. 8th Street, Federal B1dg., Rm. 867

Austin, Texas 78701

W. J. Herb, Subdistrict Chief

U.S. Geological Survey, WRD

P. 0. Box 6976; Fort Worth Federal Center

B1dg. 23, Felix and Hemphill Streets

Fort Worth, Texas 76115

R. K. Gabrysch, Subdistrict Chief

U.S. Geological Survey, WRD

2320 La Branch Street, Rm. 1112

Houston, Texas 77004
J. H. Eade, Subdistrict Chief

U.S. Geological Survey, WRD

1409 Knickerbocker Road

San Angelo, Texas 76901

C. R. Burchett, Subdistrict Chief

U.S. Geological Survey, WRD

435 I som Road

North Plaza, Suite 234

San Antonio, Texas 78216

C. C. Kidwell, Subdistrict Chief

U.S. Geological Survey, WRD

318-320A Federal B1dg.

Wichita Falls, Texas 76301

As of January 1, 1986, the surface-water data-collection network in Texas included 386 streamflow, 87 reservoir-contents, 33 stage, 10 crest-stage partialrecord, 8 periodic discharge through range, 38 flood-hydrograph partial-record, 11 flood-profile partial-record, 36 low-flow partial-record, 2 tide-level, 45 daily chemical-quality, 23 continuous-recording water-quality, 97 periodic biological, 19 lake surveys, 174 periodic organic and (or) nutrient, 4 periodic insecticide, 58 periodic pesticide, 22 automatic sampler, 157 periodic minor elements, 141 periodic chemical-quality, 108 periodic physical-organic, 14 continuous-recording three- or four-parameter water-quality, 3 sediment, 39 periodic sediment, 26 continuousrecording temperature, and 37 national stream-quality accounting network stations. Plate 1 shows the location of surface-water streamflow or reservoir content and chemical-quality or sediment stations in Texas. Plate 2 shows the location of partial-record surface-water stations. 
This index shows the station number and name, latitude and longitude, type of data collected, and the office principally responsible for the data collection (table 1). An 8-digit permanent numerical designation for gaging stations has been adopted on a nationwide basis; stations are numbered and 1 isted in downstream order. In the downstream direction along the main stem, all stations on a tributary entering above a main-stem station are listed before that station. A tributary entering between two main-stem stations is listed between them. A similar order is followed in listing stations on first rank, second rank, and other ranks of tributaries. To indicate the rank of any tributary on which a gaging station is situated and the stream to which it is an immediate tributary, each indention in the listing of gaging stations represent one rank. This downstream order and system of indention show which gaging stations are on tributaries between any two stations on a main stem and the rank of the tributary on which each gaging station is situated. On plates 1 and 2, the 8-digit station number is abbreviated because of space limitation.

\section{DESCRIPTION OF STATIONS}

CONTINUOUS-RECORDING CONDUCTIVITY STATION is a water-quality station at which a continuous record of specific conductance is obtained by a conductivity monitor. For each of these stations where discharge records are available, dischargeweighted-average concentrations and loads of selected chemical constituents are computed using the continuous record of specific conductance and regression relationships between each chemical constituent and specific conductance.

CONTINUOUS-RECORDING FOUR-PARAMETER WATER-QUALITY STATION is a water-quality station at which continuous records of specific conductance, temperature, dissolved oxygen, and $\mathrm{pH}$ are obtained by a water-quality monitor. For each of these stations where discharge records are available, discharge-weighted-average concentrations and loads of selected chemical constituents are computed using the continuous record of specific conductance and regression relationship between each chemical constituent and specific conductance.

CONTINUOUS-RECORDING TEMPERATURE STATION is a water-quality station at which a continuous record of water temperature is obtained by a temperature monitor.

DAILY CHEMICAL-QUALITY STATION is a water-quality station at which samples are collected and specific conductance and water temperature measured once daily or more frequently. For each of these stations where discharge records are available, discharge-weighted-average concentrations and loads of selected chemical constituents are computed using the daily record of specific conductance and regression relatjonship between each chemical constituent and specific conductance.

DAILY SEDIMENT STATION is a water-quality station where once daily or more frequent samples are collected and analyzed for suspended-sediment concentrations. Selected samples from most stations are analyzed for particle-size distribution. Samples of bed material also are obtained to define the size distribution of bed material available for transport. These records and records of discharge are used to compute daily discharge-weighted-average concentrations and loads of sediment transported by the flow. 
NATIONAL STREAM-QUALITY ACCOUNTING NETWORK (NASQAN) is a USGS data-collection network designed to (1) depict areal variability of streamflow and water-quality conditions nationwide on a year-by-year basis and (2) detect and assess long-term changes in streamflow and water quality.

PERIODIC BIOLOGICAL STATION is a water-quality station where samples are collected periodically (usually from 3 to 6 samples per year) and analyzed for the densities of fecal coliform and fecal streptococcal bacteria and (or) phytoplankton.

PERIODIC CHEMICAL-QUALITY STATION is a water-quality station where samples are collected periodically (usually from 2 to 10 samples per year) for the analyses of major inorganic constituents and related properties. Most of these stations on streams are operated in conjunction with a stream-gaging station; and an attempt is made to collect samples over as wide a range in discharge as possible. Some of these stations are equipped with stage-activated automatic samplers to facilitate the collection of several samples during periods of storm runoff. Samples from station on reservoirs usually are collected seasonally during comprehensive lake surveys.

PARTIAL-RECORD STATION. Because the number of streams for which information is desired far exceeds the number of stations feasible to operate for continuous record, the Geological Survey collects limited streamflow data at other streamgaging sites. When limited streamflow data are collected on a systematic basis over a period of years for use in hydrologic analyses, the site at which the data are collected is called a PARTIAL-RECORD STATION. There are four basic types of partial-record stations: (1) A flood-hydrograph partial-record station is operated for the purpose of defining stages and maximum discharges during flood periods; (2) crest-stage partial-record station is operated for the purpose of defining annual maximum stages and discharges, (3) low-flow partial-record station is operated for the purpose of defining annual minimum discharges, and (4) reconnaissance partial-record station is operated to cover a range of discharge and the corresponding variation in water quality. Discharge measurements at low-flow partial-record stations are usually made during the periods of base flow when streamflow is primarily from ground-water effluent.

PERIODIC INSECTICIDE STATION (BOTTOM SEDIMENTS) is a site where samples of bottom sediments are collected periodically (usually from 1 to 3 samples per year) and analyzed for selected organochlorine or other insecticides and related industrial compounds.

PERIODIC MINOR-ELEMENT STATION is a water-quality station where samples are collected periodically and analyzed for minor elements such as arsenic, cadmium, chromium, copper, iron, lead, manganese, mercury, selenium, silver, and zinc. Analyses of minor-element samples collected from most of the reservoir stations include only iron and manganese. The number of samples collected from stations on streams usually range from 2 to 4 per year. Reservoirs are usually sampled seasonally during comprehensive lake surveys.

PERIODIC-ORGANIC AND (OR) NUTRIENT STATION is a water-quality station where samples are collected periodically and analyzed for properties or constituents such as biochemical oxygen demand (BOD), dissolved oxygen (DO), pH, and nitrogen and phosphorus species. The number of samples varies from station to station but usually ranges from 3 to 9 samples per year. 
PERIODIC PESTICIDE STATION (WATER) is a water-quality station where samples are collected periodically (usually from 2 to 4 samples per year) and analyzed for selected pesticides such as carbonate, organochlorine, or organophosphorus insecticides and chlorophenoxy acid or triazine herbicides.

PERIODIC PHYSICAL-ORGANIC STATION is a water-quality station where samples are collected periodically and analyzed for properties or constituents such as color, turbidity, suspended solids, volatile suspended solids, and total organic carbon (TOC). The number of samples varies from station to station but usually ranges from 3 to 9 samples per year.

PERIODIC SEDIMENT STATION is a water-quality station where samples are collected periodically (usually from 4 to 9 samples per year) and analyzed for concentrations of suspended sediment. Selected samples from most station al so are analyzed for particle-size distribution. Most of these stations are operated in conjunction with a stream-gaging station, and an attempt is made to collect samples over as wide a range in discharge as possible.

At each RESERVOIR-CONTENT STATION, a daily record of stage is obtained from which a daily value of content (usually at 2400 hours) is computed, compiled, and published.

At each CONTINUOUS STREAMFLOW STATION, a permanent gage is maintained from which a continuous record of stage is obtained. Measurements of flow are made to develop stage-discharge relations. Discharge records, showing the mean daily discharge and peak discharges for the major floods of each year, and total monthly and yearly runoff expressed in inches and (or) acre-feet, are computed, compiled, and published on the basis of the water year ending September 30 .

TIDE-LEVEL STATIONS are operated along the coast to provide a continuous record of the tide level, which is used in hydrologic studies of the bays and estuaries. These records are not published.

\section{AVAILABILITY OF DATA}

Most of the information and data collected for the various types of stations listed above are computed, printed, and released in an annual publication, "Water Resources Data, Texas, Volume 1. Arkansas River Basin, Red River Basin, Sabine River Basin, Neches River Basin, Trinity River Basin, and Intervening Coastal Basins"; "Water Resources Data, Texas, Volume 2. San Jacinto River Basin, Brazos River Basin, San Bernard River Basin, and Intervening Coastal Basins"; and "Water Resources Data, Texas, Volume 3. Colorado River Basin, Lavaca River Basin, Guadalupe River Basin, Nueces River Basin, Rio Grande Basin, and Intervening Coastal Basins". These volumes are for 1 imited distribution and are published primarily for the rapid release of data shortly after the end of the water year.

The International Boundary and Water Commission, United States and Mexico, operates most streamflow and some chemcal-quality stations on the Rio Grande and near the mouths of its principal tributaries at and below El Paso, Texas. Dis- 
charge records collected at these stations are published in annual bulletins by the Commission and may be obtained by addressing your request to the office of the United States Section, Commons Bluilding C, Suite 310, 4171 N. Mesa, El Paso, Texas 79902. Some quality-of-water samples are collected by the International Boundary and Water Commission and analyzed by the Geological Survey. These records are al so published by the Geological Survey in the annual series of WATER RESOURCES DATA, TEXAS.

In addition to hydrologic information collected at established gaging stations, measurements of streamflow and water-quality data have been obtained for specific purposes at other locations in the State. Many of these measurements show the peak discharge for unusual floods, while others show minimum flows or channel losses, gains, and change in water quality in various stream reaches.

Water-supply papers and other reports containing special detailed information on flood discharges, rainfall intensities, hydrology of small watersheds, pollution by naturally occurring saltwater, and other related data have been prepared by the Geological Survey. In addition to published reports, records not yet published and file copies of special reports on floods and other investigations are available for examination in the District and Subdistrict offices (see p. 1) of the Geological Survey in Texas. 
Table 1.--Streamflow, quality, reservoir-content, and partial-record stations maintained by the U.S. Geological Survey in cooperation with State and Federal agencies.

Type of Data

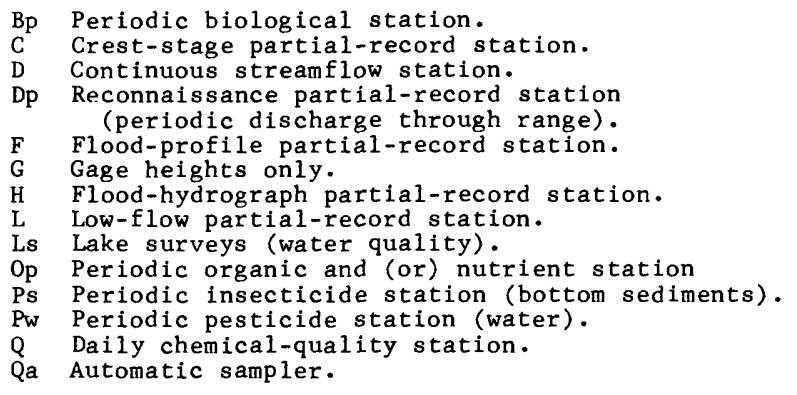

Qc Continuous-recording conductivity station.

Qm Periodic minor element station.

Qp Periodic chemical-quality station.

Qpo Periodic physical-organic station.

Qv Continuous-recording three or four-parameter water-quality station.

$R$ Reservoir-content station.

$S$ Daily sediment station.

Sp Periodic sediment station.

$\mathrm{T}$ Tide-level station.

Tc Continuous-recording temperature station

NA National accounting stream quality network (NASQAN)

() Number of discharge measurements scheduled per year.

\begin{tabular}{|c|c|c|c|c|}
\hline $\begin{array}{l}\text { Permanent } \\
\text { station } \\
\text { No. }\end{array}$ & Station & $\begin{array}{c}\text { Latitude } \\
\text { and } \\
\text { longitude }\end{array}$ & $\begin{array}{l}\text { Type } \\
\text { of } \\
\text { data }\end{array}$ & $\begin{array}{l}\text { Location } \\
\text { of } \\
r \in \text { cord a/ }\end{array}$ \\
\hline
\end{tabular}

ARKANSAS RIVER BASIN

\begin{abstract}
07227000
07227100

07227140

07227500

07227700

07227900

07227920

07228000

07235000

Canadian River at Logan, NM

Revuel to Creek near Logan, NM

Canadian River near Amarillo, TX

Chicken Creek near Amarillo, TX (2)

Lake Meredith near Sanford, TX

Dixon Creek near Borger, TX

Canadian River near Canadian, TX

North Canadian River:

Wolf Creek at Lipscomb, TX
\end{abstract}

07297910

07298100

07298200

07299540

07299570

07299670

07299750

07299840

07299890

07300000

07300500

07301300

07301410

07303300

07307700

07307800

07308200

07308500

07311669

07311700

07311782

07311783

07311800

07312000

07312100

07312110

07312200

07312500

07312700

07314000

07314500

07314800

07314900

07315200

07315500

Red River: Tule Creek.

Tule Creek near Silverton, TX Red River near Quanah, TX

Wanderers Creek at Odell, TX (2)

Salt Fork Red River:

Salt Fork Red River: Hedley, TX (2)

North Pease River:

Middle Pease River

Tongue (South Pease) River

Pease River near Childress, TX

Pease River near Vernon, TX

Red River near Burkburnett, TX

Bluff Creek: near Guthrie, TX near Guthrie, TX

Lake Kemp near Mabelle, TX

Wichita River near Mabelle, TX

Diversion Lake:

Beaver Creek near Electra, TX

Wichita River near Charlie, TX Wichita River): Red River near Terral, OK
Arkansas River:
Canadian River above New Mexico-Texas State line, NM $35^{\circ} 21^{\prime} 25^{\prime \prime} 103^{\circ} 25^{\prime} 03^{\prime \prime}$ $35^{\circ} 20^{\prime} 28^{\prime \prime}, 103^{\circ} 23^{\prime} 40^{\prime \prime}$ $35^{\circ} 23^{\prime} 35^{\prime \prime}, 103^{\circ} 02$ ' $30^{\prime \prime}$ $35^{\circ} 28^{\prime} 13^{\prime \prime}, 101^{\circ} 52^{\prime} 45^{\prime \prime}$ $35^{\circ} 28^{\prime} 29^{\prime \prime}, 101^{\circ} 45^{\prime} 35^{\prime \prime}$ $35^{\circ} 42^{\prime} 38^{\prime \prime}, 101^{\circ} 33^{\prime} 03^{\prime \prime}$ $35^{\circ} 39^{\prime} 53^{\prime \prime}, 101^{\circ} 21^{\prime} 02^{\prime \prime}$ $35^{\circ} 56^{\prime} 06^{\prime \prime}, 100^{\circ} 22^{\prime} 13^{\prime \prime}$

$36^{\circ} 14^{\prime} 19^{\prime \prime}, 100^{\circ} 16^{\prime} 31^{\prime \prime}$

$\begin{array}{lc}\text { D } & \text { b/ } \\ \text { D } & \text { b/ } \\ \text { BpOpQmQpSpNA } & \text { b/ } \\ \text { DQOpQmQpo } & \text { Wichita Falls } \\ \text { L } & \text { Do. } \\ \text { R } & \text { Do. } \\ \text { D } & \text { Do. } \\ \text { DBpOpQmQpSpNA } & \text { Do. } \\ \text { D } & \text { Do. }\end{array}$

RED RIVER BASIN

rairie Dog Town Fork Red River near Wayside, TX

MacKenzie Reservoir near Silverton, TX

Prairie Dog Town Fork Red River near Childress, TX

Groesbeck Creek at State Highway 6 near Quanah, TX

Greenbelt Lake near Clarendon, TX

Lelia Lake Creek below Bell Creek near

Salt Fork Red River near Wellington, TX

Salt Fork Red River at Mangum, OK

North Fork Red River near Shamrock, TX

Sweetwater Creek near Kelton, TX

Elm Creek near Shamrock, TX (2)

Roaring Springs near Roaring Springs, TX (1)

North Wichita River (head of Wichita River):

Truscott Brine Lake near Truscott, TX

North Wichita River near Truscott, TX

South Wichita River at Low Flow Dam

South Wichita River below Low Flow Dam

South Wichita River near Benjamin, TX

South Side Canal near Dundee, TX

Wichita River at Wichita Falls, TX

North Fork Little Wichita River (head of Little

Lake Kickapoo near Archer City, TX

Little Wichita River near Archer City, TX

Lake Arrowhead near Henrietta, TX

Little Wichita River above Henrietta, TX

East Fork Little Wichita River near Henrietta, TX $34^{\circ} 50^{\prime} 15^{\prime \prime}, 101^{\circ} 24^{\prime} 49^{\prime \prime}$

$34^{\circ} 32^{\prime} 36^{\prime \prime}, 101^{\circ} 25^{\prime} 46^{\prime \prime}$

$34^{\circ} 34^{\prime} 09^{\prime \prime}, 100^{\circ} 11^{\prime} 37^{\prime \prime}$

$34^{\circ} 24^{\prime} 47^{\prime \prime}, \quad 99^{\circ} 44^{\prime} 03^{\prime \prime}$

$34^{\circ} 21^{\prime} 16^{\prime \prime}, 99^{\circ} 44^{\prime} 24^{\prime \prime}$

$34^{\circ} 20^{\prime} 50^{\prime \prime}, 99^{\circ} 25^{\prime} 15^{\prime \prime}$

$35^{\circ} 00^{\prime} 02^{\prime \prime}, 100^{\circ} 53^{\prime} 40^{\prime \prime}$

$34^{\circ} 56^{\prime} 08^{\prime \prime}, 100^{\circ} 41^{\prime} 46^{\prime \prime}$

$34^{\circ} 57^{\prime} 27^{\prime \prime}, 100^{\circ} 13^{\prime} 14^{\prime \prime}$

$34^{\circ} 51^{\prime} 32^{\prime \prime}, \quad 99^{\circ} 30^{\prime} 28^{\prime \prime}$

$35^{\circ} 15^{\prime} 51^{\prime \prime}, 100^{\circ} 14^{\prime} 29^{\prime \prime}$

$35^{\circ} 28^{\prime} 23^{\prime \prime}, 100^{\circ} 07^{\prime} 14^{\prime \prime}$

$35^{\circ} 07^{\prime} 21^{\prime \prime}, 100^{\circ} 17^{\prime} 07^{\prime \prime}$

$33^{\circ} 51^{\prime} 12^{\prime \prime}, 100^{\circ} 51^{\prime} 53^{\prime \prime}$ $34^{\circ} 13^{\prime} 39^{\prime \prime \prime}, 100^{\circ} 04^{\prime} 24^{\prime \prime}$ $34^{\circ} 10^{\prime} 44^{\prime \prime}, 99^{\circ} 16^{\prime} 40^{\prime \prime}$

$34^{\circ} 06^{\prime} 36^{\prime \prime}, 98^{\circ} 31^{\prime} 53^{\prime \prime}$

$33^{\circ} 47^{\prime} 52^{\prime \prime}, \quad 99^{\circ} 50^{\prime} 11^{\prime \prime}$

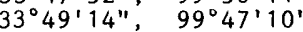

$33^{\circ} 37^{\prime} 20^{\prime \prime}$, $100^{\circ} 12^{\prime \prime} 31^{\prime \prime}$

$33^{\circ} 37^{\prime} 19^{\prime \prime}, 100^{\circ} 12^{\prime \prime} 31^{\prime \prime}$

$33^{\circ} 38^{\prime} 39^{\prime \prime}, \quad 99^{\circ} 48^{\prime} 02^{\prime \prime}$

$33^{\circ} 45^{\prime} 30^{\prime \prime}, \quad 99^{\circ} 09^{\prime} 03^{\prime \prime}$

$33^{\circ} 45^{\prime} 36^{\prime \prime}, \quad 99^{\circ} 08^{\prime} 33^{\prime \prime}$

$33^{\circ} 48^{\prime} 50^{\prime \prime}, \quad 98^{\circ} 55^{\prime} 57^{\prime \prime}$

$33^{\circ} 54^{\prime} 21^{\prime \prime}, 98^{\circ} 54^{\prime} 17^{\prime \prime}$

$33^{\circ} 54^{\prime} 34^{\prime \prime}, 98^{\circ} 32^{\prime} 00^{\prime \prime}$

$34^{\circ} 03^{\prime} 11^{\prime \prime}, 98^{\circ} 17^{\prime} 47^{\prime \prime}$

$33^{\circ} 39^{\prime} 47^{\prime \prime}, \quad 98^{\circ} 46^{\prime} 43^{\prime \prime}$

$33^{\circ} 39^{\prime} 45^{\prime \prime} \quad 98^{\circ} 36^{\prime} 46^{\prime \prime}$

$33^{\circ} 45^{\prime} 51^{\prime \prime}, \quad 98^{\circ} 22^{\prime} 17^{\prime \prime}$

$33^{\circ} 49^{\prime} 36^{\prime \prime}$ '98 $98^{\circ} 14^{\prime} 23^{\prime \prime}$

$33^{\circ} 48^{\prime} 46^{\prime \prime}, \quad 98^{\circ} 05^{\circ} 05^{\prime \prime}$

$33^{\circ} 52^{\prime} 43^{\prime \prime}, \quad 97^{\circ} 56^{\prime} 03^{\prime \prime}$ $34^{\circ} 32^{\prime} 43^{\prime \prime}, 101^{\circ} 26^{\prime} 16^{\prime \prime}$
DBpOpUmQpSpNA Wichita Fails

R Do

DBpOpQmQpSpNA

CSp Do.

$\begin{array}{ll}\mathrm{D} & \text { Do. } \\ \mathrm{L} & \text { Do }\end{array}$

$\mathrm{R}$ Do.

L Do.

DQBPOpQpo Do

$\mathrm{D}$

D Wichita

L Do.

L Do.

H

DBpOpQmQpSpNA

RQcTc

DQcSpTc

DQcTc

G Do

DQcTc Do

Do.

DQ

D Do

DQOp

$R$ Do.

D Do

R Do

D Do.

a/ Quality-of-water records are filed in the District office at Austin, Tex.

b/ Records are furnished by adjacent USGS District Offices. 
Table 1,--Streamflow, quality, reservoir-content, and partial-record stations maintained by the U.S. Geological Survey in cooperation with State and Federal agencies.--Continued

\begin{tabular}{|c|c|c|c|c|}
\hline $\begin{array}{l}\text { Permanent } \\
\text { station } \\
\text { No. }\end{array}$ & Station & $\begin{array}{c}\text { Latitude } \\
\text { and } \\
\text { longitude }\end{array}$ & $\begin{array}{l}\text { Type } \\
\text { of } \\
\text { data }\end{array}$ & $\begin{array}{c}\text { Location } \\
\text { ot } \\
\text { record a/ }\end{array}$ \\
\hline
\end{tabular}

RED RIVER BASIN--Continued

Red River:

Moss Lake near Gainesville, TX

07331500 Lake Texoma near Denison, TX

07331600

07335390

07335400

07335500

07336820

07337000

07342470

07342500

07343000

07343200

07343500

07344200

07344210

07344482

07344484

07344486

07344489

07345500

07345900

07346000

07346045

07346050

07346070

07346140

Red River at Denison Dam near Denison, TX

Pat Mayse Lake near Chicota, TX

Sanders Creek near Chicota, TX

Red River at Arthur City, TX

Red River near De Kalb, TX

Red River at Index, AR

South Sulphur River (head of Sulphur River) near Commerce, TX

South Sulphur River near Cooper, TX

North Sulphur River near Cooper, TX

Sulphur River near Talco, TX

White Oak Creek near Talco, TX

Wright Patman Lake near Texarkana, TX

Sulphur River near Texarkana, TX

Big Cypress Creek (head of Twelvemile Bayou) near Winnsboro, TX

Lake Cypress Springs near Mount Vernon, TX Brushy Creek at Scroggins, TX

Lake Bob Sandlin near Mount Pleasant, TX

Big Cypress Creek near Pittsburg, TX

Ellison Creek Reservoir near Lone Star, TX

Lake $O^{\prime}$ the Pines near Jefferson, TX

Big Cypress Creek near Jefferson, TX Black Cypress Bayou at Jefferson, TX Little Cypress Creek near Ore City, TX Little Cypress Creek near Jefferson, TX Frazier Creek near Linden, TX

$\begin{array}{lll}33^{\circ} 46^{\prime} 26^{\prime \prime}, & 97^{\circ} 12^{\prime} 50^{\prime \prime} & \mathrm{R} \\ 33^{\circ} 43^{\prime} 40^{\prime \prime}, & 97^{\circ} 09^{\prime} 35^{\prime \prime} & \mathrm{DQBpOpQmSpNA} \\ 33^{\circ} 49^{\prime} 05^{\prime \prime}, & 96^{\circ} 34^{\prime} 20^{\prime \prime} & \mathrm{R} \\ 33^{\circ} 49^{\prime}, 8^{\prime \prime}, & 96^{\circ} 33^{\prime} 47^{\prime \prime} & \mathrm{DQBpOpQmSpNA} \\ 33^{\circ} 51^{\prime} 09^{\prime \prime}, & 95^{\circ} 32^{\prime} 40^{\prime \prime} & \mathrm{R} \\ 33^{\circ} 51^{\prime} 09^{\prime \prime}, & 95^{\circ} 32^{\prime} 40^{\prime \prime} & \mathrm{D} \\ 33^{\circ} 52^{\prime} 32^{\prime \prime}, & 95^{\circ} 30^{\prime} 08^{\prime \prime} & \mathrm{D} \\ 33^{\circ} 41^{\prime} 15^{\prime \prime}, & 94^{\circ} 41^{\prime} 39^{\prime \prime} & \mathrm{DQ} 0 \mathrm{pQmQpoSp} \\ 33^{\circ} 33^{\prime} 07^{\prime \prime}, & 94^{\circ} 02^{\prime} 28^{\prime \prime} & \mathrm{D} \\ 33^{\circ} 13^{\prime} 11^{\prime \prime}, & 95^{\circ} 51^{\prime} 45^{\prime \prime} & \mathrm{D} \\ & & \\ 33^{\circ} 21^{\prime} 20^{\prime \prime}, & 95^{\circ} 35^{\prime} 39^{\prime \prime} & \mathrm{DQOpQmQpo} \\ 33^{\circ} 28^{\prime} 29^{\prime \prime}, & 95^{\circ} 35^{\prime} 15^{\prime \prime} & \mathrm{DQ} \\ 33^{\circ} 23^{\prime} 10^{\prime \prime}, & 95^{\circ} 07^{\prime} 56^{\prime \prime} & \mathrm{DQOpPsQmQpo} \\ 33^{\circ} 19^{\prime} 20^{\prime \prime}, & 95^{\circ} 05^{\prime} 33^{\prime \prime} & \mathrm{DQ} \\ 33^{\circ} 18^{\prime} 16^{\prime \prime}, & 94^{\circ} 09^{\prime} 38^{\prime \prime} & \mathrm{R} \\ 33^{\circ} 18^{\prime} 20^{\prime \prime}, & 94^{\circ} 09^{\prime} 03^{\prime \prime} & \mathrm{G} \\ 33^{\circ} 01^{\prime} 24^{\prime \prime}, & 95^{\circ} 16^{\prime} 12^{\prime \prime} & \mathrm{D} \\ & & \\ 33^{\circ} 03^{\prime} 22^{\prime \prime}, & 95^{\circ} 08^{\prime} 21^{\prime \prime} & \mathrm{R} \\ 32^{\circ} 58^{\prime} 32^{\prime \prime}, & 95^{\circ} 11^{\prime} 03^{\prime \prime} & \mathrm{D} \\ 33^{\circ} 04^{\prime} 48^{\prime \prime}, & 95^{\circ} 00^{\prime} 07^{\prime \prime} & \mathrm{R} \\ 33^{\circ} 01^{\prime} 15^{\prime \prime}, & 94^{\circ} 52^{\prime} 55^{\prime \prime} & \mathrm{DQ} \\ 32^{\circ} 55^{\prime} 16^{\prime \prime}, & 94^{\circ} 43^{\prime} 17^{\prime \prime} & \mathrm{R} \\ 32^{\circ} 45^{\prime} 04^{\prime \prime}, & 94^{\circ} 29^{\prime} 59^{\prime \prime} & \mathrm{R} \\ 32^{\circ} 44^{\prime} 58^{\prime \prime}, & 94^{\circ} 29^{\prime} 55^{\prime \prime} & \mathrm{DOP} \\ 32^{\circ} 46^{\prime} 40^{\prime \prime}, & 94^{\circ} 21^{\prime} 26^{\prime \prime} & \mathrm{D} \\ 32^{\circ} 40^{\prime} 21^{\prime \prime}, & 94^{\circ} 45^{\prime} 03^{\prime \prime} & \mathrm{D} \\ 32^{\circ} 42^{\prime} 46^{\prime \prime}, & 94^{\circ} 20^{\prime} 44^{\prime \prime} & \mathrm{DQcOpQmQpoTc} \\ 33^{\circ} 03^{\prime} 14^{\prime \prime}, & 94^{\circ} 17^{\prime} 24^{\prime \prime} & \mathrm{D} \\ & & \end{array}$

Fort Worth

c/

b/

c/

Do.

b/

Fort Worth

b/

Fort Worth

Do .

Do.

Lo.

Do.

d/

Fort Worth

Do.

Do.

Do.

Do.

Do

Fort Worth

Do.

Do.

Do.

Do.

\section{SABINE RIVER BASIN}

$33^{\circ} 07^{\prime} 58^{\prime \prime}, \quad 96^{\circ} 04^{\prime} 36^{\prime \prime}$

D Greenville, TX

08017210

08017300

08017400

08017410

08018500

08018730

08018800

08019000

08019300

08019500

08020000

08020450

08022040

08022060

08022070

08022500

08025350

08025360

08026000

08028500

08029500

08030500

08031000

Long Branch Creek near Greenville, TX

South Fork Sabine River near Quinlan, TX

Lake Tawakoni near Wills Point, TX

Sabine River near Wills Point, TX

Sabine River near Mineola, TX

Lake Fork Creek:

Burke Creek near Yantis, TX

Lake Fork Reservoir near Quitman, TX

Lake Fork Creek near Quitman, TX

Big Sandy Creek:

Lake Winnsboro near Winnsboro, TX

Big Sandy Creek near Big Sandy, TX

Sabine River near Gladewater, TX

Sabine River above Longview, Tex.

Sabine River near Beckville, TX

Martin Lake near Tatum, TX

Martin Creek near Tatum, TX

Sabine River at Logansport, LA

Toledo Bend Reservoir near Burkeville, TX

Sabine River at Toledo Bend Reservoir near Burkeville, TX

Sabine River near Burkeville, TX

abine River near Bon Wier, TX

Big Cow Creek near Newton, TX

Sabine River near Ruliff, TX

Cow Bayou near Mauriceville, TX

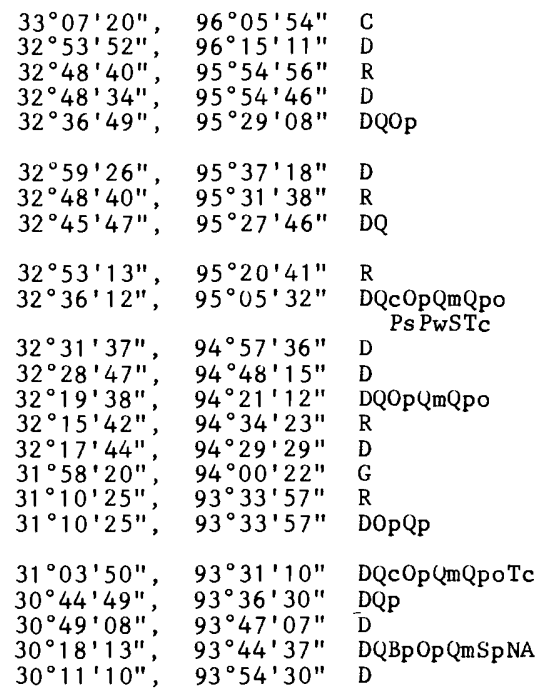

Fort Worth

Do .

Do.

Do.

Do.

Do.

Do.

Do.

Lo.

Do.

Do.

Do.

Do.

Do.

Hous ton

Do.

Do.

Do.

Do.

Do.

Do.

NECHES RIVER BASIN

Neches River

Kickapoo Creek near Brownsboro, TX

Lake Athens near Athens, TX

08031290

08031400

08032000

08033000

08033300

08033500

08033900

ake Palestine near Frankston, TX

Neches River near Neches, TX

Neches River near Dibol1, TX

Piney Creek near Groveton, TX

Neches River near Rockland, TX

Angelina River:

East Fork Angelina River near Cushing, TX Mud Creek:

Prairie Creek:

Lake Tyler near Whitehouse, TX

08034000

$\begin{array}{lll}32^{\circ} 18^{\prime} 34^{\prime \prime}, & 95^{\circ} 36^{\prime} 19^{\prime \prime} & \mathrm{D} \\ 32^{\circ} 12^{\prime} 15^{\prime \prime}, & 95^{\circ} 43^{\prime} 30^{\prime \prime} & \mathrm{R} \\ 32^{\circ} 03^{\prime} 12^{\prime \prime}, & 95^{\circ} 26^{\prime} 12^{\prime \prime} & \mathrm{R} \\ 31^{\circ} 53^{\prime} 32^{\prime \prime \prime}, & 95^{\circ} 25^{\prime} 50^{\prime \prime} & \mathrm{DQcOpTc} \\ 31^{\circ} 07^{\prime} 58^{\prime \prime}, & 94^{\circ} 48^{\prime} 35^{\prime \prime} & \mathrm{H} \\ 31^{\circ} 08^{\prime} 25^{\prime \prime}, & 95^{\circ} 05^{\prime} 1^{\prime \prime} & \mathrm{D} \\ 3^{\circ} 01^{\prime} 29^{\prime \prime}, & 94^{\circ} 23^{\prime} 55^{\prime \prime} & \mathrm{DOpQmQpQpo} \\ 31^{\circ} 51^{\prime} 36^{\prime \prime}, & 94^{\circ} 49^{\prime} 23^{\prime \prime} & \mathrm{D} \\ & & \\ 32^{\circ} 14^{\prime} 30^{\prime \prime}, & 95^{\circ} 10^{\prime} 33^{\prime \prime} & \mathrm{R}\end{array}$

Hous ton

Do.

Do.

Lo.

Do.

Do.

Do.

Do.

Do.

a/ Quality-of-water records are filed in the District Office at Austin, Tex.

b/ Records are furnished by adjacent USGS District Offices.

c/ Discharge record furnished by adjacent USGS District Offices.

d/ Discharge and/or reservoir-content records are furnished by the Corps of Engineers. 
Table 1.--Streamflow, quality, reservoir-content, and partial-record stations maintained by the U.S. Geological Survey in cooperation with State and Federal agencies.--Continued

\begin{tabular}{ccc}
$\begin{array}{c}\text { Permanent } \\
\text { station } \\
\text { No. }\end{array}$ & Station & $\begin{array}{c}\text { Latitude } \\
\text { and }\end{array}$ \\
\hline
\end{tabular}

NECHES RIVER BASIN--Continued

\begin{tabular}{|c|c|}
\hline $\begin{array}{l}08036500 \\
08036700\end{array}$ & $\begin{array}{l}\text { Angelina River near Alto, TX } \\
\text { Lake Nacogdoches near Nacogdoches, TX } \\
\text { Angelina River: }\end{array}$ \\
\hline $\begin{array}{l}08037050 \\
08038000 \\
08039100\end{array}$ & $\begin{array}{l}\text { Bayou LaNana at Nacogdoches, TX } \\
\text { Attoyac Bayou near Chireno, TX } \\
\text { Ayish Bayou near San Augustine, TX }\end{array}$ \\
\hline 08039300 & Sam Rayburn Reservoir near Jasper, TX \\
\hline & $\begin{array}{l}\text { B. A. Steinhagen Lake at Town Bluff, TX } \\
\text { Neches River at Town Bluff, TX }\end{array}$ \\
\hline 08041000 & Neches River at Evadale, TX \\
\hline $\begin{array}{l}08041500 \\
08041550\end{array}$ & $\begin{array}{c}\text { Village Creek near Kountze, TX } \\
\text { Village Creek at State Highway } 327 \text { near } \\
\text { TX (6) }\end{array}$ \\
\hline $\begin{array}{l}08041700 \\
08041720\end{array}$ & $\begin{array}{l}\text { Pine Island Bayou near Sour Lake, TX } \\
\text { Pine Island Bayou at State Highway } 105 \\
\text { Sour Lake, TX (6) }\end{array}$ \\
\hline $\begin{array}{l}08042000 \\
08042500 \\
08042510 \\
08042520\end{array}$ & $\begin{array}{l}\text { Taylor Bayou near LaBelle, TX } \\
\text { Hillebrand Bayou near Lovell Lake, TX } \\
\text { Hillebrandt Bayou at Lovell Lake, TX } \\
\text { Taylor Bayou below LaBelle, TX }\end{array}$ \\
\hline
\end{tabular}

Neches River:

Lake Nacogdoches near Nacogdoches, TX

Ayish Bayou near San Augustine, TX

Sam Rayburn Reservoir near Jasper, TX

Neches River at Town Bluff, TX

Village Creek near Kountze TX

Village Creek at State Highway 327 near Silsbee,

Pine Island Bayou near Sour Lake, TX land 105 near

\section{TAYLOR BAYOU BASIN}

$\begin{array}{ll}31^{\circ} 40^{\prime} 10^{\prime \prime}, & 94^{\circ} 57^{\prime} 24^{\prime \prime} \\ 31^{\circ} 35^{\prime} 19^{\prime \prime}, & 94^{\circ} 49^{\prime} 31^{\prime \prime} \\ 31^{\circ} 36^{\prime} 58^{\prime \prime}, & 94^{\circ} 38^{\prime} 28^{\prime \prime} \\ 31^{\circ} 30^{\prime} 15^{\prime \prime}, & 94^{\circ} 18^{\prime} 15^{\prime \prime} \\ 3^{\circ} 23^{\prime} 46^{\prime \prime}, & 94^{\circ} 09^{\prime} 03^{\prime \prime} \\ 3^{\circ} 03^{\prime} 38^{\prime \prime}, & 94^{\circ} 06^{\prime} 21^{\prime \prime} \\ 30^{\circ} 47^{\prime} 43^{\prime \prime}, & 94^{\circ} 10^{\prime} 48^{\prime \prime} \\ 30^{\circ} 47^{\prime} 36^{\prime \prime}, & 94^{\circ} 10^{\prime} 28^{\prime \prime} \\ 30^{\circ} 21^{\prime} 20^{\prime \prime}, & 94^{\circ} 05^{\prime} 35^{\prime \prime} \\ 30^{\circ} 23^{\prime} 52^{\prime \prime \prime}, & 94^{\circ} 15^{\prime} 48^{\prime \prime} \\ 30^{\circ} 20^{\prime} 48^{\prime \prime}, & 94^{\circ} 14^{\prime} 20^{\prime \prime} \\ 30^{\circ} 06^{\prime} 21^{\prime \prime}, & 94^{\circ} 20^{\prime} 04^{\prime \prime} \\ 30^{\circ} 08^{\prime} 08^{\prime \prime}, & 94^{\circ} 16^{\prime} 44^{\prime \prime}\end{array}$

\author{
D
}

D

$\mathrm{H}$

r

DUPQmQpQpo DQBPOpQmSpNA

D

DQcTc

L

Houston
Do.
Do.
Do.
Do.
d/
d/
Houston
Do.
Do.
Do.
Do.
Do.

Houston

Do.

Do

$29^{\circ} 54^{\prime} 4^{\prime \prime \prime} 99^{\circ}, 04^{\circ}, 35^{\prime \prime}, \mathrm{G}$

$29^{\circ} 52^{\prime} 55^{\prime \prime}, \quad 94^{\circ} 03^{\prime} 03^{\prime \prime}$

\section{TRINITY RIVER BASIN}

08042800

08043000

08043100

08044000

08044500

08045000

08045400

08045850

08046500

08047000

08047500

08048000

08048543

08048800

08048980

08049200

08049500

08049565

08049580

08049700

08049800

08049850

08049900

08050050

08050100

08050400

08050800

08051130

08051190

08051500

08052700

08052730

08052780

08052800

08053000

08053500

08054500

08055000

08055500

08056500

08057000

08057055

08057120

08057200

08057410

08057418

08057440
West Fork Trinity River (head of Trinity River) near Jacksboro, TX

Bridgeport Reservoir above Bridgeport, TX

West Fork Trinity River at Bridgeport, TX

Big Sandy Creek near Bridgeport, TX

West Fork Trinity River near Boyd, TX

Eagle Mountain Reservoir above Fort Worth, TX

Lake Worth above Fort Worth, TX

West Fork Trinity River:

Clear Fork Trinity River near Weatherford, TX

Benbrook Lake near Benbrook, TX

Clear Fork Trinity River near Benbrook, TX

Clear Fork Trinity River at Fort Worth, TX

West Fork Trinity River at Fort Worth, TX

West Fork Trinity River at Beach Street, Fort Worth, TX

Big Fossil Creek at Haltom City, TX

Village Creek near Everman, TX

Lake Arlington at Arlington, TX

West Fork Trinity River at Grand Prairie, TX

Big Bear Creek:

Trigg Branch at DFW Airport near Euless, TX

Mountain Creek near Venus, TX

Walnut Creek near Mansfield, TX

Joe Pool Lake near Duncanville, TX

Mountain Creek above Duncanvilie, TX

Mountain Creek near Duncanville, TX

Mountain Creek Lake near Grand Prairie, TX

Mountain Creek at Grand Prairie, TX

Elm Fork Trinity River at Gainesville, TX

Timber Creek near Collinsville, TX

Elm Fork Trinity River near Pilot Point, TX

Elm Fork Trinity River ahove Aubrey, TX

Clear Creek near Sanger, TX

Little Elm Creek near Aubrey, TX

Pecan Creek near Aubrey, TX (6)

Hickory Creek at Denton, TX

Lewisville Lake near Lewisville, TX

Elm Fork Trinity River near Lewisville, TX

Denton Creek near Justin, TX

Grapevine Lake near Grapevine. TX

Denton Creek near Grapevine, TX

E1m Fork Trinity River near Carrollton, TX Trinity River:

Turtle Creek at Dallas, TX

Trinity River at Dallas, TX

Trinity River at Cedar Crest Blvd., Dallas, TX

White Rock Creek

McKamey Creek at Preston Road, Dallas, TX

White Rock Creek at Greenville Ave., Dallas, TX

Trinity River below Dallas, TX

Fivemile Creek at Kiest Blud., Dallas, TX Newton Creek:

Whites Branch at Interstate Highway 635 , Dallas, TX $33^{\circ} 17^{\prime} 36^{\prime \prime}, \quad 98^{\circ} 04^{\prime} 43^{\prime \prime}$

$33^{\circ} 13^{\prime} 22^{\prime \prime}$,

$33^{\circ} 12^{\prime}, 07^{\prime \prime \prime}$

$33^{\circ} 13^{\prime} 54^{\prime \prime}$,

$33^{\circ} 05^{\prime} 07^{\prime \prime}$,

$32^{\circ} 52^{\prime} 39^{\prime \prime}$,
$32^{\circ} 47^{\prime} 29^{\prime \prime}$,

$32^{\circ} 44^{\prime} 25^{\prime \prime}$

$32^{\circ} 39^{\prime} 02^{\prime \prime \prime}$,

$32^{\circ} 39^{\prime} 54^{\prime \prime}$,

$32^{\circ} 43^{\prime} 56^{\prime \prime}$,
$32^{\circ} 45^{\prime} 39^{\prime \prime}$,

$32^{\circ} 45^{\prime} 06^{\prime \prime}$,

$32^{\circ} 48^{\prime} 26^{\prime \prime}$

$32^{\circ} 38^{\prime} 28^{\prime \prime}$ ',

$32^{\circ} 45^{\prime} 46^{\prime \prime}$,

$32^{\circ} 52^{\prime} 02^{\prime \prime}$,

$32^{\circ} 29^{\prime} 27^{\prime \prime}$,

$32^{\circ} 34^{\prime} 51^{\circ}$ "

$32^{\circ} 39^{\prime} 07^{\prime \prime}$ "

$32^{\circ} 39^{\prime} 43^{\prime \prime}$

$32^{\circ} 43^{\prime} 55^{\prime \prime}$

$32^{\circ} 44^{\prime} 52^{\prime \prime}$,

$33^{\circ} 37^{\prime} 27^{\prime \prime}$ "

$33^{\circ} 33^{\prime} 16^{\prime \prime}$

$33^{\circ} 21^{\prime} 01^{\prime \prime}$,

$33^{\circ} 19^{\prime} 12^{\prime \prime}$,'

$33^{\circ} 20^{\prime} 21^{\prime \prime}$

$33^{\circ} 17^{\prime} 50^{\prime \prime}$

$33^{\circ} 17^{\prime} 59^{\prime \prime}, 05^{\prime \prime}$

$33^{\circ} 04^{\prime} 09^{\prime \prime}$,

$33^{\circ} 02$ '43"',

$32^{\circ} 58^{\prime} 21^{\prime \prime \prime}$

$32^{\circ} 59^{\prime} 13^{\prime \prime}$

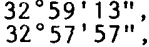

$32^{\circ} 48^{\prime} 26^{\prime \prime}$

$32^{\circ} 46^{\prime} 29^{\prime \prime}$

$32^{\circ} 45^{\prime} 04^{\prime \prime}$

$97^{\circ} 49^{\prime} 54^{\prime \prime}$

$97^{\circ} 48^{\prime} 09^{\prime \prime}$

$97^{\circ} 41^{\prime} 40^{\prime \prime}$

$97^{\circ} 33^{\prime} 30^{\prime \prime}$

$97^{\circ} 28^{\prime} 29^{\prime \prime}$

$97^{\circ} 24^{\prime} 54^{\prime \prime}$

$97^{\circ} 39^{\prime} 06^{\prime \prime}$

$97^{\circ} 26^{\prime} 54^{\prime \prime}$

$97^{\circ} 26^{\prime} 30^{\prime \prime}$

$97^{\circ} 21^{\prime} 31^{\prime}$

$97^{\circ} 19^{\prime} 56^{\prime \prime}$

$97^{\circ} 17^{\prime} 21^{\prime \prime}$

$97^{\circ} 14^{\prime} 54^{\prime \prime}$

$97^{\circ} 14^{\prime} 31^{\prime \prime}$

$97^{\circ} 11^{\prime} 32^{\prime \prime}$

$96^{\circ} 59^{\prime} 42^{\prime \prime}$

$97^{\circ} 02^{\prime} 20^{\prime \prime}$

$97^{\circ} 07^{\prime} 22^{\prime}$

$97^{\circ} 06^{\prime} \cup 6^{\prime \prime}$

$97^{\circ} 00^{\prime} 03^{\prime \prime}$

$96^{\circ} 59^{\prime} 24^{\prime \prime}$

$96^{\circ} 58^{\prime} 56^{\prime \prime}$

$96^{\circ} 56^{\prime} 35^{\prime \prime}$

$96^{\circ} 55^{\prime} 33^{\prime \prime}$

$97^{\circ} 09^{\prime} 22^{\prime \prime}$

$96^{\circ} 56^{\prime} 49^{\prime \prime}$

$97^{\circ} 02^{\prime} 49^{\prime \prime}$

$97^{\circ} 01^{\prime} 34^{\prime \prime}$

$97^{\circ} 10^{\prime} 51^{\prime \prime}$

$96^{\circ} 53^{\prime} 33^{\prime \prime}$

$96^{\circ} 55^{\prime} 06^{\prime \prime}$

$97^{\circ} 08^{\prime} 29^{\prime \prime}$

$96^{\circ} 57^{\prime} 51^{\prime \prime}$

$96^{\circ} 57^{\prime} 41^{\prime \prime}$

$97^{\circ} 031^{\prime 2} 22^{\prime \prime}$

$97^{\circ} 00^{\prime} 45^{\prime \prime}$

$97^{\circ} 00^{\prime} 45^{\prime \prime}$
$96^{\circ} 56^{\prime} 39^{\prime \prime}$

$96^{\circ} 48^{\prime} 08^{\prime \prime}$

$96^{\circ} 49^{\prime} 18^{\prime \prime}$

$96^{\circ} 47^{\prime} 07^{\prime \prime}$

$96^{\circ} 48^{\prime} 11^{\prime \prime}$

$96^{\circ} 45^{\prime} 23^{\prime \prime}$

$32^{\circ} 53^{\prime} 21^{\prime \prime}$

$32^{\circ} 42^{\prime} 26^{\prime \prime}$,

$32^{\circ} 42^{\prime} 19^{\prime \prime}$

$96^{\circ} 44^{\prime} 08^{\prime \prime}$

$96^{\circ} 51^{\prime} 32^{\prime \prime}$

$32^{\circ} 39^{\prime} 26^{\prime \prime}$

$96^{\circ} 44^{\prime} 25^{\prime \prime}$
D

R

D

R

$\mathrm{H}$

R

D

D

DOpQv

GQv

RLs OpQmQp

DOPQv

DQv

DOpQmQpQpo

RLs OpQmQp

J0pQmQpQpo

G

D

D

DOpQmQpQpo

DOpQaQmQpQpo

DOPQaQpQmQpo

DpOpQmQpQpo

DOpQaQp

RBpLsOpumQp

DQOpQmQpo

RBpLsOpQmQp

D

D

DOpQv

\section{$\mathrm{H}$}

DOpQv

$\mathrm{H}$

Wichita Falls

Fort Worth

Do.

Do.

Do.

Do.

Do

Fort Worth

Do.

Do.

Do.

Do .

Do

Do.

Do.

Do.

Do.

Do.

Do.

Do . 
Table 1.--Streamflow, quality, reservoir-content, and partial-record stations maintained by the U.S. Geological Survey in cooperation with State and Federal agencies.--Continued

\begin{tabular}{|c|c|c|c|c|}
\hline $\begin{array}{c}\text { Permanent } \\
\text { station } \\
\text { No. }\end{array}$ & Station & $\begin{array}{c}\text { Latitude } \\
\text { and } \\
\text { longitude }\end{array}$ & $\begin{array}{l}\text { Type } \\
\text { of } \\
\text { data }\end{array}$ & $\begin{array}{l}\text { Location } \\
\text { ot } \\
\text { record a/ }\end{array}$ \\
\hline
\end{tabular}

TRINITY RIVER BASIN--Continued

08057445

08058900

08059300

08059400

08059600

08060500

08061000

08061540

08061550

08061700

08061750

08062000

08062500

08062700

08062800

08062900

08063010

08063050

08063100

08063500

08063685

08063690

08063695

08063700

08063800

08064100

08064700

08064800

08065000

08065200

08065350

08065800

08066000

08066100

08066170

08066190

08066191

08066200

08066250

08066300

08066400

08066500

08067000

08067070

08067500 08067510

08067525

Trinity River:

Prairie Creek at U.S. Highway 175, Dallas, TX

East Fork Trinity River at McKinney, TX Pilot Grove Creek near Blue Ridge, TX (6) Sister Grove Creek near Blue Ridge, TX Wilson Creek at McKinney, TX (6)

Lavon Lake near Lavon, TX

East Fork Trinity River near Lavon, TX Rowlett Creek near Sachse, TX

Lake Ray Hubbard near Forney, TX Duck Creek near Garland, TX

East Fork Trinity River near Forney, TX

East Fork Trinity River near Crandall, TX

Trinity River near Rosser, TX

Trinity River at Trinidad, TX

Cedar Creek near Kemp, TX

Kings Creek near Kaufman, TX

Cedar Creek Reservoir near Trinidad, TX

Navarro Mills Lake near Dawson, TX

Richland Creek near Dawson, TX

Richland Creek near Richland, TX Chambers Creek:

Waxahachie Creek at Waxahachie, TX (6) Mustang Creek near Ennis, TX (6) Lake Clark outflow at Ennis, TX (6)

Bardwell Lake near Ennis, TX

Waxahachie Creek near Bardwe11, TX

Chambers Creek near Rice, TX

Tehuacana Creek near Streetman, TX

Catfish Creek near Tennessee Colony, TX

Trinity River near Oakwood, TX

Upper Keechi Creek near Oakwood, TX

Trinity River near Crockett, TX

Bedias Creek near Madisonville, TX

Trinity River at Riverside, TX

White Rock Creek near Trinity, TX

Kickapoo Creek near Onalaska, TX

Livingston Reservoir near Goodrich, TX

Livingston Reservoir at outflow weir near Goodrich, TX

Long King Creek at Livingston, TX

Trinity River near Goodrich, TX

Menard Creek near Rye, TX

Big Creek near Shepherd, TX

Trinity River at Romayor, TX

Trinity River at Liberty, TX

CIWA Canal near Dayton, TX

Cedar Bayou near Crosby, TX

Cedar Bayou near Baytown, TX

Goose Creek at Baytown, TX

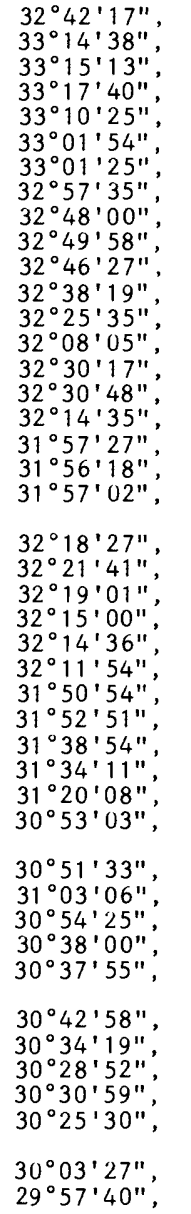

$96^{\circ} 40^{\prime} 11^{\prime \prime}$

$96^{\circ} 36^{\prime} 31^{\prime \prime}$

$96^{\circ} 24^{\prime} 4^{\prime \prime}$

$96^{\circ} 28^{\prime} 58^{\prime \prime}$

$96^{\circ} 37^{\prime} 17^{\prime \prime}$

$96^{\circ} 28^{\prime} 56^{\prime \prime}$

$96^{\circ} 28^{\prime} 31^{\prime \prime}$

$96^{\circ} 36^{\prime} 31^{\prime \prime}$

$96^{\circ} 29^{\prime} 45^{\prime \prime}$

$96^{\circ} 35^{\prime} 43^{\prime \prime}$

$96^{\circ} 30^{\prime} 12^{\prime \prime}$

$96^{\circ} 29^{\prime} 17^{\prime \prime}$

$96^{\circ} 27^{\prime} 46^{\prime \prime}$

$96^{\circ} 06^{\prime} 20^{\prime \prime}$

$96^{\circ} 06^{\prime} 55^{\prime \prime}$

$96^{\circ} 19^{\prime} 44^{\prime \prime}$

$96^{\circ} 08^{\prime} 26^{\prime \prime}$

$96^{\circ} 41^{\prime} 21^{\prime \prime}$

$96^{\circ} 25^{\prime} 16^{\prime \prime}$

$96^{\circ} 44^{\prime} 19^{\prime \prime}$

$96^{\circ} 43^{\prime} 54^{\prime \prime}$

$96^{\circ} 39^{\prime} 32^{\prime \prime}$

$96^{\circ} 38^{\prime} 49^{\prime \prime}$

$96^{\circ} 38^{\prime} 24^{\prime \prime}$

$96^{\circ} 31^{\prime} 12^{\prime \prime}$

$96^{\circ} 17^{\prime} 23^{\prime \prime}$

$95^{\circ} 52^{\prime} 07^{\prime \prime}$

$95^{\circ} 47^{\prime} 21^{\prime \prime}$

$95^{\circ} 53^{\prime} 17^{\prime \prime}$

$95^{\circ} 39^{\prime} 27^{\prime \prime}$

$95^{\circ} 46^{\prime} 39^{\prime \prime}$

$95^{\circ} 23^{\prime} 55^{\prime \prime}$

$95^{\circ} 22^{\prime} 40^{\prime \prime}$

$95^{\circ} 05^{\prime} 18^{\prime \prime}$

$95^{\circ} 00^{\prime} 36^{\prime \prime}$

$95^{\circ} 01^{\prime} 11^{\prime \prime}$

$94^{\circ} 57^{\prime} 31^{\prime \prime}$

$94^{\circ} 56^{\prime} 55^{\prime \prime}$

$94^{\circ} 46^{\prime} 46^{\prime \prime}$

$94^{\circ} 59^{\prime} 06^{\prime \prime}$

$94^{\circ} 51^{\prime} 02^{\prime \prime}$

$\mathrm{D}$

DOpQmQpQpo

DpOpQmQpQpo

UpQmQpQpo

POpQmQpQpo

R

D

DQOPQmQPoQv

DOpQv

BBPOpQmupQvSpNA

D

$\mathrm{R}$

R

DQOpQmQpo

Fort Worth

Do.

Do.

Do.

Do.

d/

Fort Worth

Do.

Do.

Do.

Do.

Do.

Do.

Do.

Do.

d/

Dp Op QmQpQpo

DpOpQmQpQpo

DpOpQmQpQpo

DQUpQmQpo

$\mathrm{D}$
$\mathrm{D}$

D

DOpQmQpoQv

$\mathrm{DQcQmQpQpo}$

PwSTC

$G$

D

RLs UpQmQp

D

DQP

DQBp Op Pw

$94^{\circ} 49^{\prime} 05^{\prime \prime}$
$94^{\circ} 48^{\prime} 36^{\prime \prime}$

QaQmQpoNA

D

Fort wo
Do.

Do.

Do.

Do.

d/

ort Wor
Do.

Do.

Houston

Do.

Do .

Do.

Do.

Do.

Do.

Do.

Do.

Do.

Do.

Do.

Do.

Do.

Do.

CEDAR BAYOU BASIN

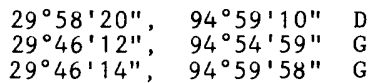

Hous ton

Do.

SAN JACINTO RIVER BASIN

West Fork San Jacinto River (head of San Jacinto River):

08067600

08067610

08067650

08067900

08068000

08068090

08068325

08068400

08068438

08068450

08068520

08068700

08068720

08068740

08068780

08068800

08068900

08069000

Lake Conroe near Conroe, TX

Lake Conroe at outflow weir near Conroe, TX

West Fork San Jacinto River below Lake Conroe near Conroe, TX

Lake Creek near Conroe, TX (12 e/)

West Fork San Jacinto River near Conroe, TX

West Fork San Jacinto River above Lake Houston near Porter, TX

Spring Creek:

Willow Creek near Tomball, TX

Panther Branch near Conroe, TX

Swale No. 8 at Woodlands, TX

Panther Branch near Spring, TX

Spring Creek at Spring, TX

Cypress Creek at Sharp Road near Hockley, TX Cypress Creek at Katy-Hockley Road near Hockley, TX

Cypress Creek at House-Hah1 Road near Cypress, TX

Little Cypress Creek near Houston, TX

Cypress Creek at Grant Road near Houston, TX

Cypress Creek at Stuebner-Airline Road near Westfield, TX

Cypress Creek near Westfield, TX

$30^{\circ} 21 ' 30^{\prime \prime}$,

$95^{\circ} 33^{\prime} 39^{\prime \prime}$

$95^{\circ} 33^{\prime}, 37^{\prime \prime}$

RLs OpQmQp

(1)

$30^{\circ} 20^{\prime} 31^{\prime \prime}, \quad 95^{\circ} 32$ '34"

DOp PwQmQpQpo

Do .

$30^{\circ} 15^{\prime} 12^{\prime \prime}, \quad 95^{\circ} 34^{\prime} 43^{\prime \prime} \quad$ Dp OpQmQpQpoPs Pw

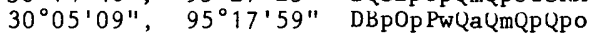

Do.

Do.

$30^{\circ} 06^{\prime} 19^{\prime \prime}$,

$30^{\circ} 11^{\prime} 28^{\prime \prime}$

$30^{\circ} 08^{\prime} 38^{\prime \prime}$

$30^{\circ} 08^{\prime} 02^{\prime \prime}$

G

$95^{\circ} 28^{\prime} 44^{\prime \prime} \mathrm{H}$

$95^{\circ} 28^{\prime} 09^{\prime \prime}$

$95^{\circ} 28^{\prime} 38^{\prime \prime}$

$95^{\circ} 24^{\prime} 21^{\prime \prime}$

$\mathrm{H}$

$29^{\circ} 55^{\prime} 15^{\prime \prime}, \quad 95^{\circ} 50^{\prime} 24^{\prime \prime}$

$29^{\circ} 57^{\prime} 00^{\prime \prime}, \quad 95^{\circ} 48^{\prime} 29^{\prime \prime}$ 
Table 1.--Streamflow, quality, reservoir-content, and partial-record stations maintained by the U.S. Geological Survey in cooperation with State and Federal agencies.--Continued

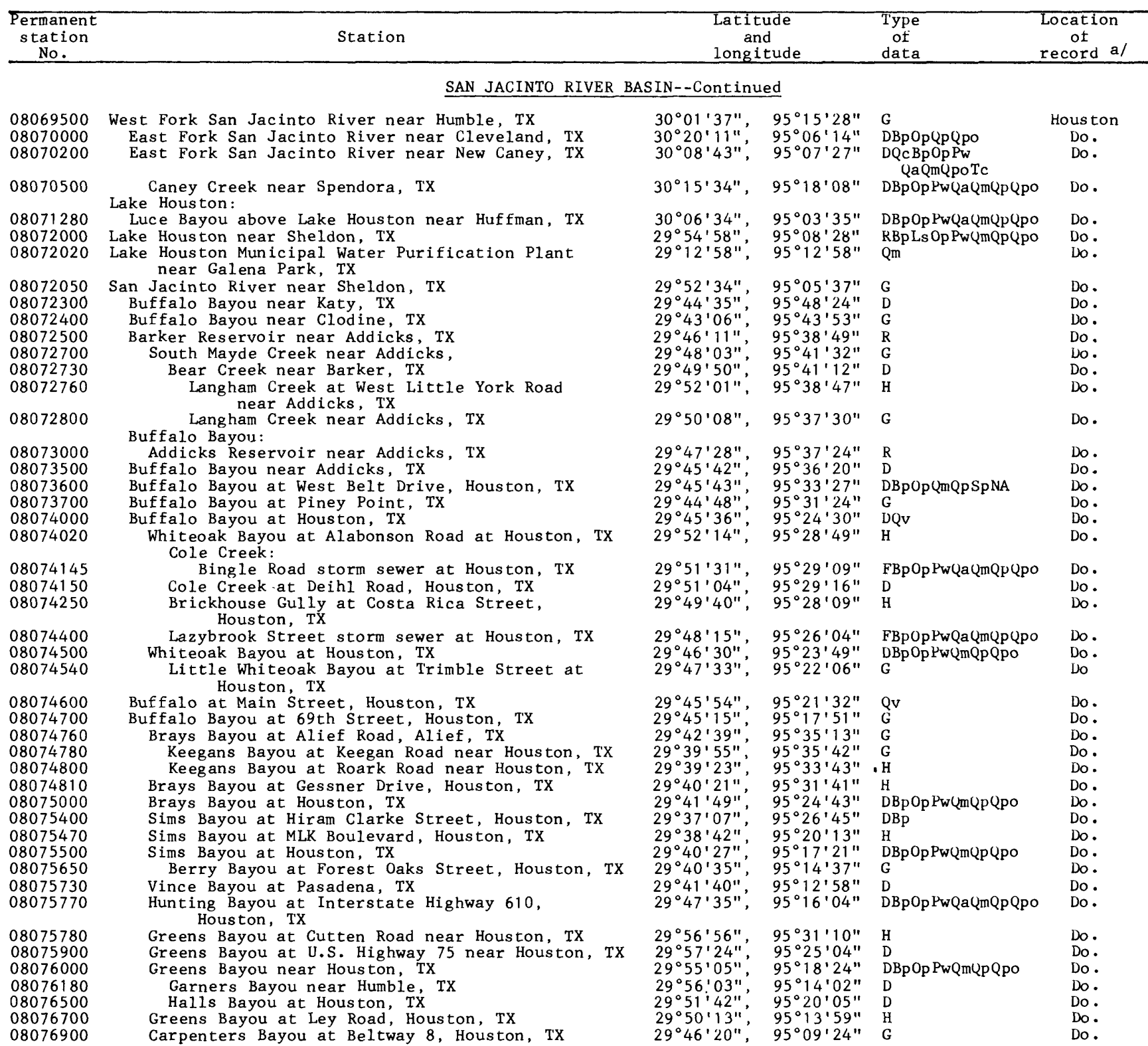

\section{CLEAR CREEK BASIN}

08077000 Clear Creek near Pearland, TX Turkey Creek:

Beamer Ditch at Houston, T'X

08077505

08077520

08077600

08077630
Turkey Creek near Friendswood, TX
Clear Creek near Friendswood, TX

Chigger Creek:

Armand Bayou (formerly Middle Bayou):

Horsepen Bayou at Bay Area Blvd, Houston. TX $29^{\circ} 35^{\prime} 50^{\prime \prime}, \quad 95^{\circ} 17^{\prime} 11^{\prime \prime}$ D

$29^{\circ} 35^{\prime} 30^{\prime \prime}, \quad 95^{\circ} 13^{\prime} 19^{\prime \prime} \quad G$

$29^{\circ} 35^{\prime} 02^{\prime \prime}, 95^{\circ} 11^{\prime} 13^{\prime \prime}$

$29^{\circ} 31^{\prime} 02^{\prime \prime}$, 95०10.42" C

$29^{\circ} 35^{\prime} 00^{\prime \prime}, \quad 95^{\circ} 06^{\prime} 12^{\prime \prime} \quad \mathrm{G}$

$29^{\circ} 26^{\prime} 50^{\prime \prime}, \quad 94^{\circ} 55^{\prime} 12^{\prime \prime} \quad$ G

Hous ton

Hous ton

Do.

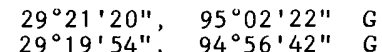

08077780 Highland Bayou near Texas City, TX

CHOCOLATE BAYOU BASIN
Hous ton

Do

Do.

Do.
08077650 Moses Lake-Galveston Bay near Texas City, TX

\section{HIGHLAND BAYOU BASIN}

08078000 Chocolate Bayou near Alvin, TX

$29^{\circ} 22^{\prime} 09^{\prime \prime}, \quad 95^{\circ} 19^{\prime} 14^{\prime \prime} \quad$ D

Houston 
Table 1.--Streamflow, quality. reservoir-content and partial-record stations maintained by the U.S. Geological Survey in cooperation with State and Federal agencies.--Continued

\begin{tabular}{ccc}
\hline $\begin{array}{c}\text { Permanent } \\
\text { station } \\
\text { No. }\end{array}$ & Station & $\begin{array}{c}\text { Latitude } \\
\text { and } \\
\text { longitude }\end{array}$ \\
\hline
\end{tabular}

\section{BRAZOS RIVER BASIN}

Double Mountain Fork Brazos River:

Blackwater Draw (head of North Fork Double Mountain Fork Brazos River):

08079300 08079575

Blackwater Draw tributary near Floyd, NM

North Fork Double Mountain Fork Brazos River near Post, TX

08079600 Double Mountain Fork Brazos River at Justiceburg, TX

08080500

08080600

08080900

08080950

08081000

08081200

08082000

08082180

08082500

08082700

08082800

08083100

08083240

08083245

08083500

08084000

08084100

08084500

08084800

08085500

08086150

08086212

08086290

08086400

08086500

08088000

08088300

08088400

08088450

08088500

08088600

08089000

08090800

08090900

08091000

08091500

08091730

08091750

08092000

08092500

08092600

08093100

08093160

08093250

08093260

08093350

08093360

08093500

08094800

08095000

08095200

08095300

08095400

08095550

08096500

08098290

08099100

08099300

08099400

08099500

08100000

Salt Mountain Fork Brazos

Running Water Draw near Clovis, NM

White River below falls near Crosbyton, TX (4)

Duck Creek near Girard, TX

Salt Fork Brazos River near Peacock, TX

Croton Creek near Jayton, TX

Salt Fork Brazos River near Aspermont, TX

Brazos River:

North Croton Creek near Knox City, TX

Brazos River at Seymour, TX

Millers Creek near Munday, TX

Millers Creek Reservoir near Bomarton, TX

Clear Fork Brazos River near Roby, TX

Clear Fork Brazos River at Hawley, TX

Mulberry Creek near Hawley, IXX

Clear Fork Brazos River:

Fort Phantom Hill Reservoir near Nugent, TX

Clear Fork Brazos River at Nugent, TX

Deadman Creek near Nugent, TX (6 e $/$ )

Paint Creek:

Lake Stamford near Haske11, TX

Paint Creek:

California Creek near Stamford, TX

Clear Fork Brazos River at Fort Griffin, TX Hubbard Creek:

Salt Prong Hubhard Creek:

North Fork Hubbard Creek near Albany, TX

Hubbard Creek below Albany, TX

Big Sandy Creek above Breckenridge, TX

Hubbard Creek Reservoir near Breckenridge, TX

Hubbard Creek near Breckenridge, TX

Brazos River near South Bend, TX

Salt Creek:

Briar Creek near Graham TX

Lake Graham near Graham, TX

Big Cedar Creek near Ivan, TX

Possum Kingdom Lake near Graford, TX

Brazos River at Morris Sheppard Dam near Graford, TX

Brazos River near Palo Pinto, TX

Brazcs River near Dennis, TX

Lake Granbury near Granbury, TX

razos River near Glen Rose, TX

Paluxy River at Glen Rose, TX

Squaw Creek:

Squaw Creek Reservoir near Glen Rose, TX Squaw Creek near Glen Rose, TX

Nolan River at Blum. TX

Lake Whitney near Whitney, TX

Brazos River at Whitney Dam near Whitney, TX

razos River near Aquilla, TX

Aquil.la Creek near Peoria, TX (9 e/)

Hackberry Creek at Hillsboro, $T \overline{\mathrm{X}}$

Hackberry Creek below Hillsboro, TX (9)

Aquilla Lake above Aquilla, TX

Aquilla Creek above Aquilla, TX

Aquilla Creek near Aquilla, TX

North Bosque River at Hico, TX

North Bosque River near Clifton, T

North Bosque River at Valley Mills, TX South Bosque River:

Middle Bosque River near McGregor, TX Hog Creek near Crawford, TX

Waco Lake near Waco, TX

Brazos River at Waco, TX

Brazos River near Highbank, TX

Leon River (head of Little River) near De Leon, TX

Sabana River near De Leon, TX

Proctor Lake near Proctor, TX

Leon River near Hasse, TX

Leon River near Hamilton, TX

\begin{tabular}{|c|c|c|c|}
\hline $\begin{array}{l}34^{\circ} 13^{\prime} 13^{\prime \prime}, \\
33^{\circ} 14^{\prime} 52^{\prime} \text {, }\end{array}$ & $\begin{array}{l}103^{\circ} 45^{\circ} 05^{\prime \prime} \\
101^{\circ} 20^{\prime} 24^{\prime \prime}\end{array}$ & $\begin{array}{l}\mathrm{C} \\
\mathrm{DQ}\end{array}$ & San Angelo \\
\hline $\begin{array}{l}33^{\circ} 02^{\prime} 18^{\prime \prime}, \\
33^{\circ} 00^{\prime} 29^{\prime \prime},\end{array}$ & $\begin{array}{l}101^{\circ} 11^{\prime} 50^{\prime \prime} \\
100^{\circ} 10^{\prime} 49^{\prime \prime}\end{array}$ & $\begin{array}{l}\text { DQ } \\
\text { DQBpUpQmSpNA }\end{array}$ & $\begin{array}{l}\text { Do. } \\
\text { Do. }\end{array}$ \\
\hline $\begin{array}{l}34^{\circ} 31^{\prime} 56^{\prime \prime}, \\
33^{\circ} 39^{\prime} 57^{\prime \prime}, \\
33^{\circ} 21^{\prime} \cdot 22^{\prime \prime}, \\
33^{\circ} 12,43^{\prime \prime}, \\
33^{\circ} 17^{\prime} 18^{\prime \prime}, \\
33^{\circ} 20^{\prime} 02^{\prime \prime},\end{array}$ & $\begin{array}{l}103^{\circ} 12^{\prime} 05^{\prime \prime} \\
101^{\circ} 09^{\prime} 35^{\prime \prime} \\
100^{\circ} 42^{\prime} 17^{\prime \prime} \\
100^{\circ} 25^{\prime} 53^{\prime \prime} \\
100^{\circ} 25^{\prime} 5^{\prime \prime} \\
100^{\circ} 14^{\prime} 16^{\prime \prime}\end{array}$ & $\begin{array}{l}\text { C } \\
L \\
D \\
\text { DQ } \\
\text { DQcTc } \\
\text { DBpUpQmQpSpNA }\end{array}$ & 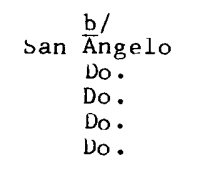 \\
\hline $\begin{array}{l}33^{\circ} 22^{\prime} 59^{\prime \prime}, \\
33^{\circ} 34^{\prime} 51^{\prime \prime}, \\
33^{\circ} 19^{\prime} 45^{\prime \prime}, \\
33^{\circ} 24^{\prime} 32^{\prime \prime}, \\
32^{\circ} 47^{\prime} 15^{\prime \prime}, \\
32^{\circ} 35^{\prime} 53^{\prime \prime}, \\
32^{\circ} 34^{\prime} 04^{\prime \prime},\end{array}$ & $\begin{array}{r}100^{\circ} 04^{\prime} 51^{\prime \prime} \\
99^{\circ} 16^{\prime} 02^{\prime \prime} \\
99^{\circ} 27^{\prime}, 33^{\prime \prime} \\
99^{\circ} 23^{\prime} 19^{\prime \prime} \\
100^{\circ} 23^{\prime} 18^{\prime \prime} \\
99^{\circ} 48^{\prime} 53^{\prime \prime} \\
99^{\circ} 47^{\prime} 32^{\prime \prime}\end{array}$ & $\begin{array}{l}\mathrm{DQ} \\
\mathrm{DQ} \\
\mathrm{D} \\
\mathrm{R} \\
\mathrm{D} \\
\mathrm{D} \\
\mathrm{D}\end{array}$ & $\begin{array}{l}\text { Do. } \\
\text { Wichita Fatls } \\
\text { Do. } \\
\text { Do. } \\
\text { San Angelo } \\
\text { Do. } \\
\text { Do. }\end{array}$ \\
\hline $\begin{array}{l}32^{\circ} 36^{\prime} 58^{\prime \prime}, \\
32^{\circ} 41^{\prime} 24^{\prime \prime}, \\
32^{\circ} 40^{\prime} 36^{\prime \prime},\end{array}$ & $\begin{array}{l}99^{\circ} 40^{\prime} 05^{\prime \prime} \\
99^{\circ} 40^{\prime} 09^{\prime \prime} \\
99^{\circ} 37^{\prime} 00^{\prime \prime}\end{array}$ & $\begin{array}{l}\text { R } \\
\mathrm{D} \\
\mathrm{LOpQp}\end{array}$ & $\begin{array}{l}\text { Do. } \\
\text { Do. } \\
\text { Do. }\end{array}$ \\
\hline $33^{\circ} 04^{\prime} 44^{\prime \prime}$, & $99^{\circ} 34^{\prime} 52^{\prime \prime}$ & K & Wichita falls \\
\hline $\begin{array}{l}32^{\circ} 55^{\prime} 51^{\prime \prime}, \\
32^{\circ} 56^{\prime} 04^{\prime \prime},\end{array}$ & $\begin{array}{l}99^{\circ} 38^{\prime} 32^{\prime \prime} \\
99^{\circ} 13^{\prime} 27^{\prime \prime}\end{array}$ & $\begin{array}{l}\mathrm{D} \\
\mathrm{D}\end{array}$ & $\begin{array}{l}\text { Do. } \\
\text { Do. }\end{array}$ \\
\hline $\begin{array}{l}32^{\circ} 42^{\prime} 27^{\prime \prime}, \\
32^{\circ} 43^{\prime} 58^{\prime \prime}, \\
32^{\circ} 38^{\prime} 54^{\prime \prime}, \\
32^{\circ} 49^{\prime} 53^{\prime \prime}, \\
32^{\circ} 50^{\prime} 13^{\prime \prime}, \\
33^{\circ} 01^{\prime} 27^{\prime \prime},\end{array}$ & $\begin{array}{l}99^{\circ} 16^{\prime} 29^{\prime \prime} \\
99^{\circ} 08^{\prime} 25^{\prime \prime} \\
99^{\circ} 00^{\prime} 15^{\prime \prime} \\
98^{\circ} 58^{\prime} 03^{\prime \prime} \\
98^{\circ} 56^{\prime} 52^{\prime \prime} \\
98^{\circ} 38^{\prime} 37^{\prime \prime}\end{array}$ & $\begin{array}{l}\text { DQ } \\
\text { DQcTc } \\
\text { DQcTc } \\
\text { RLs UpUmQp } \\
\text { D } \\
\text { DBpOpQmQpSpNA }\end{array}$ & $\begin{array}{c}\text { San Angelo } \\
\text { Do. } \\
\text { Do. } \\
\text { Do. } \\
\text { Do. } \\
\text { Wichita Falls }\end{array}$ \\
\hline $\begin{array}{l}33^{\circ} 12^{\prime} 43^{\prime \prime}, \\
33^{\circ} 08^{\prime} 04^{\prime \prime}, \\
32^{\circ} 49^{\prime} 39^{\prime \prime}, \\
32^{\circ} 52 \text { ' } 20^{\prime \prime}, \\
32^{\circ} 52^{\prime} 00^{\prime \prime},\end{array}$ & $\begin{array}{l}98^{\circ} 37^{\prime} 06^{\prime \prime} \\
98^{\circ} 36^{\prime} 48^{\prime \prime} \\
98^{\circ} 43^{\prime} 25^{\prime \prime} \\
98^{\circ} 25^{\prime} 32^{\prime \prime} \\
98^{\circ} 26^{\prime} 00^{\prime \prime}\end{array}$ & $\begin{array}{l}D \\
R \\
D \\
R L s \cup p Q m Q p \\
Q\end{array}$ & $\begin{array}{l}\text { Do. } \\
\text { Do. } \\
\text { Do. } \\
\text { Do. }\end{array}$ \\
\hline $\begin{array}{l}32^{\circ} 51^{\prime} 45^{\prime \prime}, \\
32^{\circ} 36^{\prime} 56^{\prime \prime}, \\
32^{\circ} 22^{\prime} 27^{\prime \prime}, \\
32^{\circ} 16^{\prime} 18^{\prime \prime \prime}, \\
32^{\circ} 13^{\prime} 53^{\prime \prime},\end{array}$ & $\begin{array}{l}98^{\circ} 18^{\prime} 08^{\prime \prime} \\
97^{\circ} 55^{\prime} 32^{\prime \prime} \\
97^{\circ} 41^{\prime} 20^{\prime \prime} \\
97^{\circ} 39^{\prime} 48^{\prime \prime} \\
97^{\circ} 46^{\prime} 37^{\prime \prime}\end{array}$ & $\begin{array}{l}\text { D } \\
D Q \\
\text { RLsOpQmQp } \\
\text { DOpQmQpQuo } \\
\text { H }\end{array}$ & $\begin{array}{l}\text { Do. } \\
\text { Fort Worth } \\
\text { Do. } \\
\text { Do. } \\
\text { Do. }\end{array}$ \\
\hline 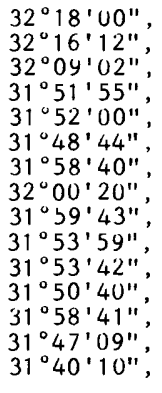 & $\begin{array}{l}97^{\circ} 47^{\prime} 12^{\prime \prime} \\
97^{\circ} 43^{\prime} 56^{\prime \prime} \\
97^{\circ} 24^{\prime} 0 y^{\prime \prime} \\
97^{\circ} 22^{\prime} 18^{\prime \prime} \\
97^{\circ} 22^{\prime} 00^{\prime \prime} \\
97^{\circ} 17^{\prime} 51^{\prime \prime} \\
97^{\circ} 14^{\prime} 44^{\prime \prime} \\
97^{\circ} 08^{\prime} 59^{\prime \prime} \\
97^{\circ} 08^{\prime} 38^{\prime \prime} \\
97^{\circ} 12^{\prime} 09^{\prime \prime} \\
97^{\circ} 12^{\prime} 21^{\prime \prime \prime} \\
97^{\circ} 12^{\prime} 04^{\prime \prime} \\
97^{\circ} 02^{\prime} 04^{\prime \prime} \\
97^{\circ} 34^{\prime} 04^{\prime \prime} \\
97^{\circ} 28^{\prime} 09^{\prime \prime}\end{array}$ & $\begin{array}{l}\text { R } \\
D \\
H \\
\text { KBpLs OpQmQp } \\
Q \\
D \\
\text { LpOpQmUpQpo } \\
\text { DOpQmQpQpo } \\
\text { LOpQmQpQpo } \\
\text { RBpLsUpQmQp } \\
\text { D } \\
\text { DOpQmQpQpo } \\
\text { D } \\
\text { D } \\
\text { D }\end{array}$ & $\begin{array}{c}\text { Do. } \\
\text { Do. } \\
\text { Do. } \\
\text { d/ } \\
\text { Fort Worth } \\
\text { Do. } \\
\text { Do. } \\
\text { Do. } \\
\text { Do. } \\
\text { Do. } \\
\text { Do. } \\
\text { Do. } \\
\text { Austin } \\
\text { Do. }\end{array}$ \\
\hline $\begin{array}{l}31^{\circ} 30 \cdot 33^{\prime \prime}, \\
31^{\circ} 33^{\prime} 20^{\prime \prime}, \\
31^{\circ} 34^{\prime} 46^{\prime \prime}, \\
31^{\circ} 32^{\prime} 06^{\prime \prime}, \\
31^{\circ} 08^{\prime} 02^{\prime \prime}, \\
32^{\circ} 10^{\prime} 25^{\prime \prime},\end{array}$ & $\begin{array}{l}97^{\circ} 21^{\prime} 56^{\prime \prime} \\
97^{\circ} 21^{\prime} 22^{\prime \prime} \\
97^{\circ} 11^{\prime} 51^{\prime \prime} \\
97^{\circ} 04^{\prime} 22^{\prime \prime} \\
96^{\circ} 49^{\prime} 29^{\prime \prime} \\
98^{\circ} 31^{\prime} 58^{\prime \prime}\end{array}$ & $\begin{array}{l}\mathrm{H} \\
\mathrm{H} \\
\mathrm{R} \\
\mathrm{D} \\
\mathrm{DQ} \mathrm{CBPOp}\left(\mathrm{mSp} \mathrm{P}^{\mathrm{S}} \mathrm{NA}\right. \\
\mathrm{H}\end{array}$ & $\begin{array}{c}\text { Do. } \\
\text { Do. } \\
\text { d/ } \\
\text { Austin } \\
\text { Do. } \\
\text { San Angelo }\end{array}$ \\
\hline $\begin{array}{l}32^{\circ} 06^{\prime} 50^{\prime \prime}, \\
31^{\circ} 58^{\prime} 07^{\prime \prime}, \\
31^{\circ} 57^{\prime} 28^{\prime \prime}, \\
31^{\circ} 47^{\prime} 19^{\prime \prime},\end{array}$ & $\begin{array}{l}98^{\circ} 36^{\prime} 19^{\prime \prime} \\
98^{\circ} 29^{\prime} 09^{\prime \prime} \\
98^{\circ} 27^{\prime} 32^{\prime \prime} \\
98^{\circ} 07^{\prime} 16^{\prime \prime}\end{array}$ & $\begin{array}{l}H \\
R \\
D \\
D\end{array}$ & $\begin{array}{c}\text { Do. } \\
\text { d/ } \\
\text { San Ange Lo } \\
\text { Austin }\end{array}$ \\
\hline
\end{tabular}

a/ Quality-of-water records are filed in the District Office at Austin, I'ex.

b/ Records are furnished by adjacent USGS District Oftices.

d/ Discharge and/or reservoir-content records are furnished by the Corps of Engineers.

e/ Water samples for chemical analysis are collected to cover the range in stage and discharge. 
Table 1.- Streamflow, quality, reservoir-content, and partial-record stations maintained by the U.S. Geological Survey in cooperation with State and Federal agencies.--Continued

\begin{tabular}{|c|c|c|c|c|}
\hline $\begin{array}{c}\text { Permanent } \\
\text { station } \\
\text { No. }\end{array}$ & Station & $\begin{array}{c}\text { Latitude } \\
\text { and } \\
\text { longitude }\end{array}$ & $\begin{array}{l}\text { Type } \\
\text { of } \\
\text { data }\end{array}$ & $\begin{array}{l}\text { Location } \\
\text { of } \\
\text { record a/ }\end{array}$ \\
\hline
\end{tabular}

BRAZOS RIVER BASIN--Continued

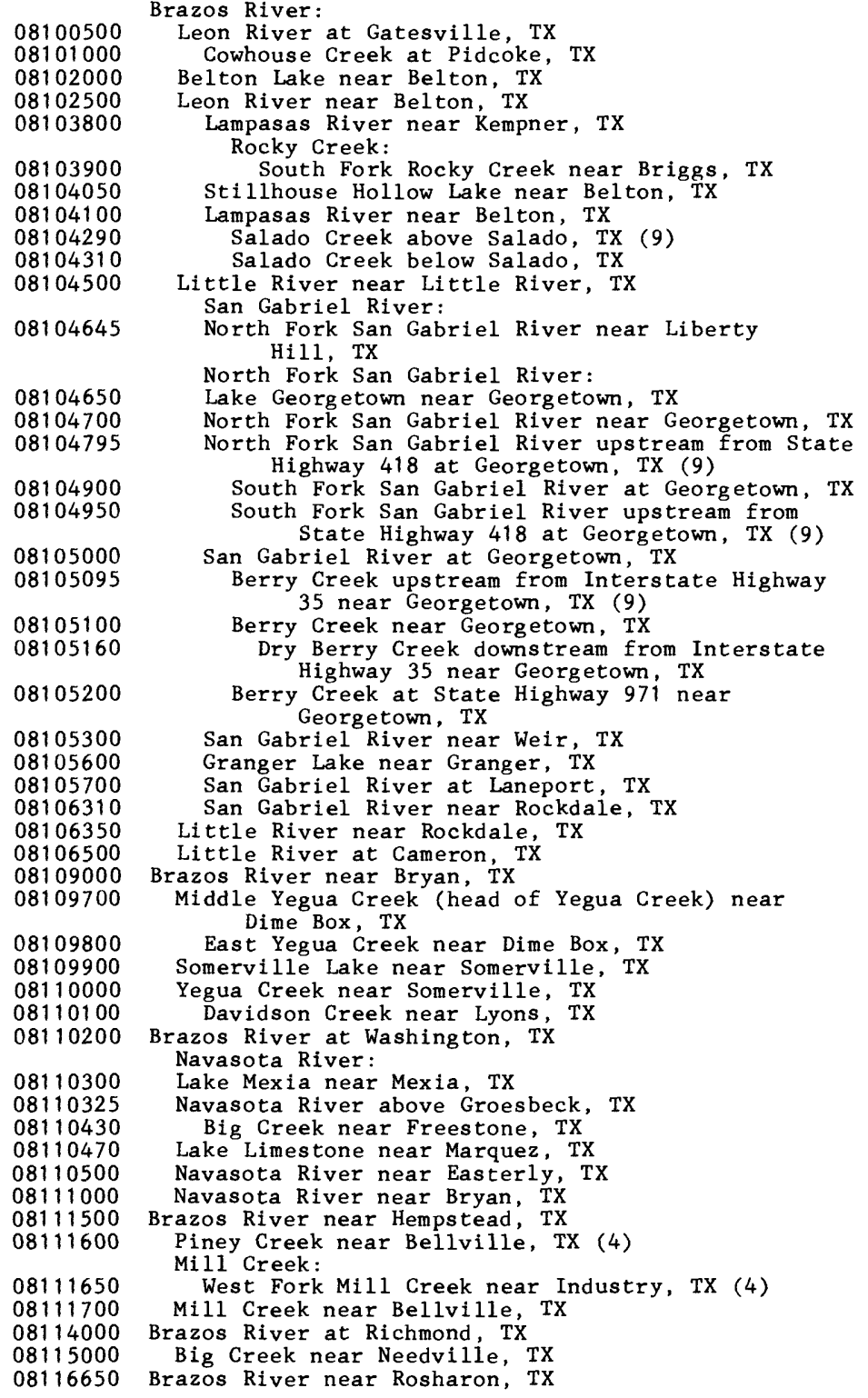

\begin{tabular}{|c|c|c|c|}
\hline $\begin{array}{l}31^{\circ} 25^{\prime} 58^{\prime \prime}, \\
31^{\circ} 17^{\prime} 05^{\prime \prime}, \\
31^{\circ} 066^{\prime} 22^{\prime \prime}, \\
31^{\circ} 04^{\prime} 12^{\prime \prime \prime}, \\
31^{\circ} 04^{\prime} 54^{\prime \prime},\end{array}$ & $\begin{array}{l}97^{\circ} 45^{\prime} 42^{\prime \prime} \\
97^{\circ} 53^{\prime}, 05^{\prime \prime} \\
97^{\circ} 28^{\prime} 28^{\prime \prime} \\
97^{\circ} 26^{\prime} 28^{\prime \prime} \\
98^{\circ} 00^{\prime} 59^{\prime \prime}\end{array}$ & $\begin{array}{l}D \\
D \\
R \\
D \\
D\end{array}$ & $\begin{array}{l}\text { Austin } \\
\text { Do. } \\
\text { d/ } \\
\text { Austin } \\
\text { Do. }\end{array}$ \\
\hline $\begin{array}{l}30^{\circ} 54 \text { '41",', } \\
31^{\circ} 01^{\prime} 20^{\prime \prime}, \\
31^{\circ} 00^{\prime} 06 ", \\
30^{\circ} 56^{\prime} 42^{\prime \prime \prime}, \\
30^{\circ} 57^{\prime} 07 ", \\
30^{\circ} 57^{\prime} 59^{\prime \prime},\end{array}$ & $\begin{array}{l}98^{\circ} 02,12 " \prime \\
97^{\circ} 31,57^{\prime \prime} \\
97^{\circ} 29,32 " 1 \\
97^{\circ} 32,30^{\prime \prime} \\
97^{\circ} 31,26^{\prime \prime} \\
97^{\circ} 20^{\prime} 45^{\prime \prime}\end{array}$ & $\begin{array}{l}\text { DBpOpQmQpSp } \\
R \\
D \\
L \\
L \\
D\end{array}$ & $\begin{array}{l}\text { Do. } \\
\text { d/ } \\
\text { Austin } \\
\text { Do. } \\
\text { Do. } \\
\text { Do. }\end{array}$ \\
\hline $30^{\circ} 42 ' 11^{\prime \prime}$, & $97^{\circ} 52^{\prime} 37^{\prime \prime}$ & OpQmQpQpo & \\
\hline $\begin{array}{l}30^{\circ} 40^{\prime} 03^{\prime \prime}, \\
30^{\circ} 39^{\prime} 42^{\prime \prime}, \\
30^{\circ} 38^{\prime} 44^{\prime \prime},\end{array}$ & $\begin{array}{l}97^{\circ} 43^{\prime} 38^{\prime \prime} \\
97^{\circ} 42^{\prime} 40^{\prime \prime} \\
97^{\circ} 40^{\prime} 49^{\prime \prime}\end{array}$ & $\begin{array}{l}\text { RBp Ls 0pQmQp } \\
\text { DOpQmQpQpo } \\
\text { L }\end{array}$ & $\begin{array}{c}\text { d/ } / \\
\text { Austin } \\
\text { Do. }\end{array}$ \\
\hline $\begin{array}{l}30^{\circ} 37^{\prime} 32 ", \text { ', } \\
30^{\circ} 38^{\prime} 38^{\prime \prime},\end{array}$ & $\begin{array}{l}97^{\circ} 41^{\prime} 27^{\prime \prime} \\
97^{\circ} 40^{\prime} 50^{\prime \prime}\end{array}$ & $\begin{array}{l}\text { D } \\
\text { L }\end{array}$ & $\begin{array}{l}\text { Do. } \\
\text { Do. }\end{array}$ \\
\hline $\begin{array}{l}30^{\circ} 39^{\prime} 14^{\prime \prime}, \\
30^{\circ} 42^{\prime} 11^{\prime \prime},\end{array}$ & $\begin{array}{l}97^{\circ} 39^{\prime} 18^{\prime \prime} \\
97^{\circ} 39^{\prime} 58^{\prime \prime}\end{array}$ & $\begin{array}{l}\mathrm{L} \\
\mathrm{L}\end{array}$ & $\begin{array}{l}\text { Do. } \\
\text { Do. }\end{array}$ \\
\hline $\begin{array}{l}30^{\circ} 41 \text { ' } 28 \text { ", }^{\circ}, \\
30^{\circ} 41 \text { ' } 04^{\prime \prime},\end{array}$ & $\begin{array}{l}97^{\circ} 39^{\prime} 21^{\prime \prime} \\
97^{\circ} 38^{\prime} 14^{\prime \prime}\end{array}$ & $\begin{array}{l}\text { D } \\
\text { L }\end{array}$ & $\begin{array}{l}\text { Do. } \\
\text { Do. }\end{array}$ \\
\hline $30^{\circ} 40^{\prime} 33^{\prime \prime}$, & $97^{\circ} 36^{\prime} 51^{\prime \prime}$ & $\mathrm{L}$ & Do. \\
\hline 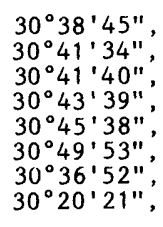 & $\begin{array}{l}97^{\circ} 35^{\prime} 06^{\prime \prime} \\
97^{\circ} 19^{\prime} 34^{\prime \prime} \\
97^{\circ} 16^{\prime} 43^{\prime \prime} \\
97^{\circ} 02^{\prime} 19^{\prime \prime} \\
97^{\circ} 00^{\prime} 49^{\prime \prime} \\
96^{\circ} 57^{\prime} 01^{\prime \prime} \\
96^{\circ} 29^{\prime} 10^{\prime \prime} \\
96^{\circ} 54^{\prime} 16^{\prime \prime}\end{array}$ & $\begin{array}{l}\text { DOpQmQpQpo } \\
\text { RBpLsOpQmQp } \\
\text { DOpQmQpQpo } \\
\text { D } \\
\text { D } \\
\text { DQBpOpQmSpNA } \\
\text { D } \\
\text { D }\end{array}$ & $\begin{array}{c}\text { Do. } \\
\text { d/ } \\
\text { Austin } \\
\text { Do. } \\
\text { Do. } \\
\text { Do. } \\
\text { Do: } \\
\text { Do. }\end{array}$ \\
\hline $\begin{array}{l}30^{\circ} 24^{\prime} \\
30^{\circ} 16^{\prime \prime} 6^{\prime \prime}, \\
30^{\circ} 19^{\prime} 18^{\prime \prime}, \\
30^{\circ} 25^{\prime} 10^{\prime \prime}, \\
30^{\circ} 21^{\prime} \cdot 40^{\prime \prime},\end{array}$ & $\begin{array}{l}96^{\circ} 49^{\prime} 02^{\prime \prime} \\
96^{\circ} 31^{\prime} 32^{\prime \prime} \\
96^{\circ} 30^{\prime} 26^{\prime \prime} \\
96^{\circ} 32^{\prime} \cdot 24^{\prime \prime} \\
96^{\circ} 09^{\prime} 18^{\prime \prime}\end{array}$ & $\begin{array}{l}\text { DOpQmQpQpo } \\
\text { RBpLsOpQmQp } \\
\text { DOpQmQpQpo } \\
\text { D } \\
\text { G }\end{array}$ & $\begin{array}{c}\text { Do. } \\
\text { d } / \\
\text { Austin } \\
\text { Do. } \\
\text { Do. }\end{array}$ \\
\hline $\begin{array}{l}31^{\circ} 38^{\prime} 37^{\prime \prime}, \\
31^{\circ} 34^{\prime} \cdot 27^{\prime \prime}, \\
31^{\circ} 30^{\prime} 25^{\prime \prime}, \\
31^{\circ} 19^{\prime} 30^{\prime \prime \prime}, \\
31^{\circ} 10^{\prime} 12^{\prime \prime \prime}, \\
30^{\circ} 52,10^{\prime \prime}, \\
30^{\circ} 07^{\prime} 44^{\prime \prime \prime}, \\
29^{\circ} 57^{\prime} 06^{\prime \prime},\end{array}$ & $\begin{array}{l}96^{\circ} 34^{\prime} 43^{\prime \prime} \\
96^{\circ} 31^{\prime} 14^{\prime \prime} \\
96^{\circ} 19^{\prime} 31^{\prime \prime} \\
96^{\circ} 19^{\prime} 08^{\prime \prime} \\
96^{\circ} 17^{\prime} 51^{\prime \prime} \\
96^{\circ} 11^{\prime} 32^{\prime \prime} \\
96^{\circ} 11^{\prime} 15^{\prime \prime} \\
96^{\circ} 10^{\prime} 20^{\prime \prime}\end{array}$ & $\begin{array}{l}\text { R } \\
\text { DQ } \\
D \\
\text { RLs OpQmQp } \\
\text { D } \\
\text { D } \\
\text { D } \\
\text { L }\end{array}$ & $\begin{array}{l}\text { Do. } \\
\text { Do. } \\
\text { Do. } \\
\text { Do. } \\
\text { Do. } \\
\text { Do. } \\
\text { Houston } \\
\text { Do. }\end{array}$ \\
\hline $\begin{array}{l}29^{\circ} 58^{\prime} 55^{\prime \prime}, \\
29^{\circ} 52,51^{\prime \prime}, \\
29^{\circ} 34^{\prime} 56^{\prime \prime}, \\
29^{\circ} 28^{\prime} 35^{\prime \prime}, \\
29^{\circ} 50^{\prime} 58^{\prime \prime},\end{array}$ & $\begin{array}{l}96^{\circ} 30^{\prime} 00^{\prime \prime} \\
96^{\circ} 12^{\prime} 18^{\prime \prime} \\
95^{\circ} 45^{\prime} 27^{\prime \prime \prime} \\
95^{\circ} 48^{\prime} 4^{\prime \prime} \\
95^{\circ} 34^{\prime} 56^{\prime \prime}\end{array}$ & $\begin{array}{l}\text { L } \\
\text { D } \\
\text { DQBPOpQmSNA } \\
\text { D } \\
\text { D }\end{array}$ & $\begin{array}{l}\text { Do. } \\
\text { Do. } \\
\text { Do. } \\
\text { Do. } \\
\text { Do. }\end{array}$ \\
\hline
\end{tabular}

SAN BERNARD RIVER BASIN

$29^{\circ} 18^{\prime} 47^{\prime \prime}, \quad 95^{\circ} 53^{\prime} 36^{\prime \prime}$ DBpOpQmQpSpNA Houston

08117500 San Bernard River near Boling, TX COLORADO RIVER BASIN

08118000

08119500

08120500

08120700

08121000

08123000

08123600

08123720

08123800

08123850

08123950

08124000
Colorado River:

Lake J. B. Thomas near Vincent, TX

Colorado River near Ira, TX

Deep Creek near Dunn, TX

Colorado River near Cuthbert, TX

Colorado River at Colorado City, TX

Morgan Creek:

Lake Colorado City near Colorado City, TX

Champion Creek:

Champion Creek Reservoir near Colorado City, TX

Beals Creek near Coahoma, TX

Beals Creek near Westbrook, TX

Colorado River above Silver, TX

E. V. Spence Reservoir near Robert Lee, TX

Colorado River at Robert Lee, TX

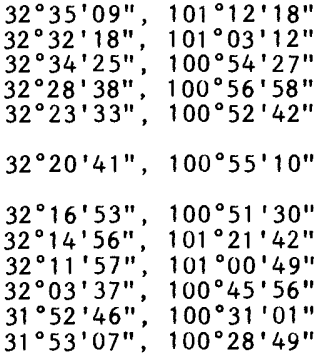

$\mathrm{K}$

D

DQcTc

$D Q$

$R$

$\mathrm{DQCTC}$

QQcTc

DQcBPOpUmSpTcNA

RLs OPQmQP

$\mathrm{D}$
San Angelo

Do.

Do.

Do.

Do.

Do.

Do.

Do.

Do.

Do.

a' Quality-of-water records are filed in the District Office at Austin. Tex.

d/ Discharge and/or reservoir-content records are furnished by the Corps of Engineers. 
Table 1.--Streamflow, quality, reservoir-content, and partial-record stations maintained by the U.S. Geological Survey in cooperation with State and Federal agencies.--Continued

\begin{tabular}{ccc}
\hline $\begin{array}{c}\text { Permanent } \\
\text { station } \\
\text { No. }\end{array}$ & Station & $\begin{array}{c}\text { Latitude } \\
\text { and } \\
\text { longitude }\end{array}$ \\
\hline
\end{tabular}

COLORADO RIVER BASIN--Continued

08126380 Colorado River near Ballinger, TX

08127000

08128000

08128400

08129300

08129500

08130500

08131200

08131400

08132000

08133500

08134000

08134500

08135000

08136000

08136500

08136700

08138000

08141000

08141500

08142000

08143000

08143600

08143900

08144500

08144600

08145000

08146000

08146500

08147000

08148000

08149400

08149500

08150000

08150700

08150800

08151500

08152000

08152900

08153500

08154500

08154510

08154700

08154900

08155260

08155300

08155400

08155500

08156800

08157900 08158000 08158050

08158100 08158200 08158300 08158380 08158600 08158640

08158650 08158700 08158810

08158840 08158880

Elm Creek at Ballinger, TX at Christoval, TX Dove Creek: TX (9)

South Concho River:

Concho River at San Angelo, TX

Concho River at Paint Rock, TX

Colorado River near Stacy, TX

Colorado River at Winche11, TX

Pecan Bayou:

Jim Ned Creek:

Hords Creek

Hords Creek near Valera, TX

Hords Creek at Coleman, TX

Pecan Bayou near Mullin, TX

San Saba River:

San Saba River at Menard, TX

San Saba River near Brady, TX

Brady Creek at Brady, TX

San Saba River at San Saba, TX

Colorado River near San Saba, TX

Lake Buchanan near Burnet, TX

North Llano River:

Colorado River:

North Llano River

South Llano River:

Llano River near Junction, TX

Llano River near Mason, TX

Beaver Creek near Mason, TX

Llano River at Llano, TX

Sandy Creek near Kingsland, TX

Lake Travis near Austin, TX

Lake Austin at Austin, TX

Colorado River (Town Lake) :

Barton Springs at Austin, TX

Town Lake at Austin, TX

Colorado River at Austin, TX Austin, TX $(12 \mathrm{e} /)$

Colorado River below Austin, IX

nion Creek near Driftwood, TX Driftwood, TX
South Concho River (head of Concho River)

Middle Concho River above Tankersley, TX

Spring Creek above Tankersley, TX

Dove Creek Spring near Knickerbocker,

Dove Creek at Knickerbocker, TX

Twin Buttes Reservoir near San Angelo, TX

Pecan Creek near San Angelo, TX

Lake Nasworthy near San Angelo, TX

North Concho River at Sterling City, TX

North Concho River near Carlsbad, TX

0 . C. Fisher Lake at San Ange10, TX

North Concho River at San Angelo, TX

Hords Creek Lake near Valera, TX

Lake Brownwood near Brownwood, TX

Springs at Fort McKavett, TX (2)

San Saba Springs at San Saba, TX (2)

South Llano River near Telegraph, TX (2)

Seven Hundred Springs near Telegraph, TX (2)

Pedernales River near Fredericksburg, $T X$

Pedernales River near Johnson City, TX

Colorado River below Mansfield Dam, Austin, TX

Bul1 Creek at Loop 360 near Austin, TX

Barton Creek near Camp Craft Road near Austin, TX

Barton Creek at Loop 360, Austin, TX

Barton Creek above Barton Springs, Austin, TX (4)

Shoal Creek at 12 th Street, Austin, TX

Boggy Creek at U.S. Highway 183, Austin, TX

Walnut Creek at Farm Road 1325 near Austin, TX

Walnut Creek at Dessau Road, Austin, TX

Ferguson Branch at Springdale Road, Austin, TX

Little Walnut Creek at Georgian Drive, Austin, TX

Walnut Creek at Webberville Road, Austin, TX

Walnut Creek at Southern Pacific Railroad Bridge

Bear Creek below Farm Road 1826 near

Slaughter Creek at Farm Road 1826 near Austin, TX Boggy Creek (south) at Circle S Road, Austin, TX

$31^{\circ} 42^{\prime} 55^{\prime \prime}, 100^{\circ} 01^{\prime} 34^{\prime \prime}$

年

$31^{\circ} 11^{\prime} 16^{\prime \prime}, 100^{\circ} 30^{\prime} 09^{\prime \prime}$

DQ

$31^{\circ} 25^{\prime} 38^{\prime \prime}, \quad 100^{\circ} 42^{\prime} 39^{\prime \prime}$

$31^{\circ} 19^{\prime} 48^{\prime \prime}, 100^{\circ} 38^{\prime} 24^{\prime \prime}$

D

$31^{\circ} 11^{\prime} 06^{\prime \prime}, 100^{\circ} 43^{\prime} 51^{\prime \prime}$

$31^{\circ} 22^{\prime} 55^{\prime \prime}, 100^{\circ} 37^{\prime}, 45^{\prime \prime}$

$31^{\circ} 18^{\prime} 32^{\prime \prime}, 100^{\circ} 26^{\prime} 44^{\prime \prime}$

$31^{\circ} 23^{\prime} 19^{\prime \prime}, 100^{\circ} 28^{\prime} 41^{\prime \prime}$

$31^{\circ} 49^{\prime} 48^{\prime \prime}, 100^{\circ} 59^{\prime} 36^{\prime \prime}$

$31^{\circ} 35^{\prime} 33^{\prime \prime}, 100^{\circ} 38^{\prime} 12^{\prime \prime}$

$31^{\circ} 29^{\prime} 04^{\prime \prime}, 100^{\circ} 28^{\prime} 53^{\prime \prime}$

$31^{\circ} 27^{\prime} 57^{\prime \prime}, 100^{\circ} 26^{\prime} 51^{\prime \prime}$

$31^{\circ} 27^{\prime} 16^{\prime \prime}, 100^{\circ} 24^{\prime} 37^{\prime \prime}$

$31^{\circ} 30^{\prime} 57^{\prime \prime}, 99^{\circ} 55^{\prime} 09^{\prime \prime}$

$31^{\circ} 29^{\prime} 37^{\prime \prime}, \quad 99^{\circ} 34^{\prime} 25^{\prime \prime}$

$31^{\circ} 28^{\prime} 04^{\prime \prime}$, 9909'43" D record a/

$\begin{array}{ll}31^{\circ} 49^{\prime} 58^{\prime \prime}, & 99^{\circ} 33^{\prime} 38^{\prime \prime} \\ 31^{\circ} 50^{\prime} 04^{\prime \prime}, & 99^{\circ} 33^{\prime} 26^{\prime \prime}\end{array}$

$31^{\circ} 50^{\prime} 50^{\prime \prime}, \quad 99^{\circ} 25^{\prime} 25^{\prime \prime}$

$31^{\circ} 50^{\prime} 13^{\prime \prime}, 99^{\circ} 00^{\prime} 13^{\prime \prime}$

$31^{\circ} 31^{\prime} 02^{\prime \prime}, 98^{\circ} 44^{\prime} 25^{\prime \prime}$ DQ

$30^{\circ} 50^{\prime} 03^{\prime \prime}, 100^{\circ} 05^{\prime} 37^{\prime \prime}$

$30^{\circ} 55^{\prime} 08^{\prime \prime}, \quad 99^{\circ} 47^{\prime} 07^{\prime \prime}$

$31^{\circ} 00^{\prime} 11^{\prime \prime}, \quad 99^{\circ} 16^{\prime} 07^{\prime \prime}$

$31^{\circ} 08^{\prime} 17^{\prime \prime}, \quad 99^{\circ} 20^{\prime} 05^{\prime \prime}$

$31^{\circ} 12^{\prime} 47^{\prime \prime}, 98^{\circ} 43^{\prime} 09^{\prime \prime}$

$31^{\circ} 11^{\prime} 44^{\prime \prime}, 98^{\circ} 42^{\prime} 42^{\prime \prime}$

$31^{\circ} 13^{\prime} 04^{\prime \prime}, \quad 98^{\circ} 33^{\prime} 51^{\prime \prime}$

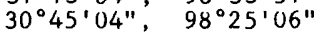

$30^{\circ} 15^{\prime} 43^{\prime \prime}, \quad 99^{\circ} 56^{\prime} 01^{\prime \prime}$

San Angelo

Do.

Do.

Do.

D Do.

Do.

Do.

Do.

Do.

d/

DoOpQmupo

QQ

Do.

Do.

Do.

Do.

d/

San Angelo

Lo.

Do.

Do.

Do .

Do.

Do.

Do .

Do.

Do.

Do.

$30^{\circ} 16^{\prime} 12^{\prime \prime}$,

$30^{\circ} 29^{\prime} 45^{\prime \prime}$

$30^{\circ} 39^{\prime} 35^{\prime \prime}$

$30^{\circ} 38^{\prime} 39^{\prime \prime}$

$30^{\circ} 45^{\prime} 04^{\prime \prime}$

$30^{\circ} 13^{\prime} 13^{\prime \prime}$

$30^{\circ} 17$ ' $30^{\prime \prime}$

$30^{\circ} 23^{\prime} 29^{\prime \prime}$

$30^{\circ} 23^{\prime} 30^{\prime \prime}$

$30^{\circ} 22$ '19",

$99^{\circ} 55^{\prime} 22^{\prime \prime}$

$99^{\circ} 43$ '19"

99.06'29"

$99^{\circ} 05^{\prime} 46^{\prime \prime}$

$98^{\circ} 40^{\prime} 10^{\prime \prime}$

$98^{\circ} 28^{\prime} 19^{\prime \prime}$

$98^{\circ} 52^{\prime} 10^{\prime \prime}$

$98^{\circ} 23^{\prime} 57^{\prime \prime}$

$97^{\circ} 54^{\prime} 24^{\prime \prime}$

$97^{\circ} 54^{\prime} 28^{\prime \prime}$

$97^{\circ} 47^{\prime} 04^{\prime \prime}$

$30^{\circ} 18^{\prime} 53^{\prime \prime}$,

$97^{\circ} 47^{\prime} 10^{\prime \prime}$

$30^{\circ} 16^{\prime} 12^{\prime \prime}, \quad 97^{\circ} 49^{\prime} 43^{\prime \prime}$

$30^{\circ} 14^{\prime} 40^{\prime \prime}, \quad 97^{\circ} 48^{\prime} 07^{\prime \prime}$

$30^{\circ} 15^{\prime} 48^{\prime \prime}, \quad 97^{\circ} 46^{\prime} 19^{\prime \prime}$

$30^{\circ} 15^{\prime} 48^{\prime \prime}, \quad 97^{\circ} 46^{\prime} 16^{\prime \prime}$

$30^{\circ} 16^{\prime} 35^{\prime \prime}$

$97^{\circ} 45^{\prime} 00^{\prime \prime}$

$30^{\circ} 14^{\prime} 56^{\prime \prime}$

$30^{\circ} 14^{\prime} 40^{\prime \prime}$ ',

$97^{\circ} 43^{\prime} 03^{\prime \prime}$

$97^{\circ} 41^{\prime} 39^{\prime \prime}$

$97^{\circ} 40^{\prime} 20^{\prime \prime}$

$30^{\circ} 24^{\prime} 35^{\prime \prime}$,
$30^{\circ} 22^{\circ} 30^{\prime \prime}$,

$97^{\circ} 42^{\prime} 41^{\prime \prime}$
$97^{\circ} 39^{\prime} 37^{\prime \prime}$

$30^{\circ} 19^{\prime} 54^{\prime \prime}$

$97^{\circ} 39^{\prime} 13^{\prime \prime}$

$97^{\circ} 41^{\prime} 52^{\prime \prime}$

$97^{\circ} 39^{\prime} 17^{\prime \prime}$

$30^{\circ} 15^{\prime} 58^{\prime \prime}, \quad 97^{\circ} 39^{\prime} 24^{\prime \prime}$

$30^{\circ} 12^{\prime} 28^{\prime \prime}, \quad 97^{\circ} 38^{\prime} 15^{\prime \prime}$

$30^{\circ} 04^{\prime} 59^{\prime \prime}, \quad 98^{\circ} 00^{\prime} 29^{\prime \prime}$
$30^{\circ} 09^{\prime}, 9^{\prime \prime}, \quad 97^{\circ} 56^{\prime} 23^{\prime \prime}$

$97^{\circ} 56^{\prime} 23^{\prime \prime}$

$30^{\circ} 12^{\prime} 32^{\prime \prime}$

$97^{\circ} 54^{\prime} 11$ ' $97^{\circ} 46^{\prime} 55^{\prime \prime}$

L
D
D
D
DBpOpQmUpSpNA
D

Do .

Do.

עo.

Do .

San Angelo

Aust in

Do.

Do.

DOpQp

DBpOpPw

QaUmQpQpo

BpLsOpPwQmQpQpo

DBpOpPwQa

QmQpQpo

DBpOpHwQa

$\mathrm{L}$

DBpOp PwQmQpQpo

BBPOpPwQ

UmupQpo

BpLsOp PwQmQpQpo

DQBpOpQmSpNA

DBpOpPwQa

$\mathrm{H}$

HBpOp PwQmQpQpo

$\mathrm{H}$

DBpOp PwQmQpQpo

LBpOp PwQmQpQpo

Bp0p PwQmQpQpo 
Table 1 --Streamflow, quality, reservoir-content and partial-record stations maintained by the U.S. Geological Survey in cooperation with State and Federal agencies.--Continued

\begin{tabular}{|c|c|c|c|c|}
\hline $\begin{array}{l}\text { Permanent } \\
\text { station } \\
\text { No. }\end{array}$ & Station & $\begin{array}{c}\text { Latitude } \\
\text { and } \\
\text { longitude }\end{array}$ & $\begin{array}{l}\text { Type } \\
\text { of } \\
\text { data }\end{array}$ & $\begin{array}{l}\text { Location } \\
\text { of } \\
\text { record a/ }\end{array}$ \\
\hline
\end{tabular}

COLORADO RIVER BASIN--Continued

Colorado River (Town Lake):

Onion Creek:

08158920 Williamson Creek at Oak Hill, TX

08158930

08158970

08159000

08159200

08160800

08161000

08162000

Williamson Creek at Manchaca Road, Austin, TX

Williamson Creek at Jimmy Clay Road, Austin, TX

Onion Creek at U.S. Highway 183, Austin, TX

Colorado River at Bastrop, TX

Cummins Creek:

Redgate Creek near Columbus, TX

Colorado River at Columbus, TX
Colorado River at Wharton, TX

Colorado River near Bay City, TX $30^{\circ} 14^{\prime} 06^{\prime \prime}$,
$30^{\circ} 13$ '16",
$30^{\circ} 11 \cdot 21^{\prime \prime}$,
$30^{\circ} 10^{\prime} 40^{\prime \prime}$,
$30^{\circ} 06^{\prime} 20^{\prime \prime}$,
$29^{\circ} 47^{\prime} 56^{\prime \prime}$,
$29^{\circ} 42$ ' $22^{\prime \prime}$,
$29^{\circ} 18^{\prime} 32^{\prime \prime}$,
$28^{\circ} 58^{\prime} 26^{\prime \prime}$,

$97^{\circ} 51^{\prime} 36^{\prime \prime}$

$97^{\circ} 47^{\prime} 36^{\prime \prime}$

$97^{\circ} 43^{\prime} 56^{\prime \prime}$

$97^{\circ} 19^{\prime} 08^{\prime \prime}$

$96^{\circ} 31^{\prime} 55^{\prime \prime}$

$96^{\circ} 32^{\prime} 12^{\prime \prime}$

$96^{\circ} 06^{\prime} 13^{\prime \prime}$
DBpOpPwQa

QmQpQpo

DBpOp PwQmQpQpo

DBpOpPwQmQpQpo

DOpQp

D

$\begin{array}{ll}\text { D } & \text { Housto } \\ \text { D } & \text { Do. }\end{array}$

DQBp OpQmQpoSpNA Do.

D

\section{TRES PALACIOS RIVER BASIN}

08162600 Tres Palacios River near Midfield, TX

$28^{\circ} 55^{\prime} 40^{\prime \prime}, \quad 96^{\circ} 10^{\prime} 15^{\prime \prime}$

San Antonio

\section{LAVACA RIVER BASIN}

08163500 Lavaca River at Hallettsville, TX

08164000 Lavaca River near Edna, TX

08164300 Navidad River near Hallettsville, TX

08164350

08164450

08164503

Navidad River near Speaks, TX

Sandy Creek near Louise, TX

Mustang Creek:

West Mustang Creek near Ganado, TX

GARCITAS CREEK BASIN

08164600 Garcitas Creek near Inez, TX

$29^{\circ} 26^{\prime} 35^{\prime \prime}$,

$28^{\circ} 57^{\prime} 35^{\prime \prime}$

$29^{\circ} 19^{\prime} 18^{\prime \prime}$

$29^{\circ} 09^{\prime} 34^{\prime \prime}$

$29^{\circ} 04^{\prime} 17^{\prime \prime}$

$96^{\circ} 56^{\prime} 39^{\prime \prime}$

$96^{\circ} 41^{\prime} 10^{\prime \prime}$

$96^{\circ} 48^{\prime} 45^{\prime \prime}$

$96^{\circ} 42^{\prime} 32^{\prime \prime}$

$96^{\circ} 32^{\prime} 47^{\prime \prime}$

$96^{\circ} 28^{\prime} 01^{\prime \prime}$

$\mathrm{D}$

DBpOpQmQp SpNA

$\mathrm{D}$

DOpQmQpQpo

DOpQmQpQpo

$28^{\circ} 53^{\prime} 28^{\prime \prime}, \quad 96^{\circ} 49^{\prime} 08^{\prime \prime} \quad \mathrm{DOpQmQpQpo}$

San Antonio

PLACEDO CREEK BASIN

08164800 Placedo Creek near Placedo, TX

$28^{\circ} 43^{\prime} 30^{\prime \prime}, \quad 96^{\circ} 46^{\prime} 07^{\prime \prime}$

D

San Antonio

GUADALUPE RIVER BASIN
08165300

08165500

08166000

08167000

08167500

08167700
08167800

08167800

08168500

08168600

08168700

08168800

08169000

08169500

08169580

08170000

08171000

08171300

08172000

08172400

08173000

08173900

08175000

08175800

08176500

08176550

08176900

08176990

08177240

08177300

08177360

08177380

08177400

08177500
08166140

08177410
Guadalupe River:

North Fork Guadalupe River near Hunt, TX

Guadalupe River at Hunt, TX

Johnson Creek near Ingram, TX

Guadalupe River above Bear Creek at Kerrville, TX

Guadalupe River at Comfort, TX

Guadalupe River near Spring Branch, TX

Canyon Lake near New Braunfels, TX

Guadalupe River at Sattler, TX

Hueco Springs near New Braunfels, TX (9)

Guadalupe River above Comal River at New Braunfels, TX

Comal River:

Blieders Creek at New Braunfels, TX (2)

Panther Canyon at New Braunfels, TX (2)

Dry Comal Creek at New Braunfels, TX (2)

Comal River at New Braunfels, TX

Guadalupe River at New Braunfeis, TX

Guadalupe River below New Braunfels, TX

San Marcos River spring flow at San Marcos, TX

Blanco River at Wimberley, TX

Blanco River near Kyle, TX

San Marcos River at Luling, TX

Plum Creek at Lockhart, TX

Plum Creek near Luling, TX

Guadalupe River at Gonzales, TX

Sandies Creek near Westhoff, TX

Guadalupe River at Cuero, TX

Guadalupe River at Victoria, TX

Coleto Creek:

Fifteenmile Creek near Wesser, TX

Coleto Creek at Arnold Road Crossing near Schroeder, TX

Coleto Creek Reservoir inflow (Guadalupe Diversion) near Schroeder, TX

Coleto Creek Reservoir (Turkey Creek arm) near Schroeder, TX

Perdido Creek at Farm Road 622 near Fannin, TX

Coleto Creek Reservoir (condenser No. 1) near Fannin, TX

Coleto Creek Reservoir (Sulphur Creek arm) near Fannin, TX

Coleto Creek Reservoir near Victoria, TX

Coleto Creek Reservoir (outflow) near Victoria, TX

Coleto Creek near Victoria, TX $30^{\circ} 03^{\prime} 36^{\prime \prime}$,

30 $30^{\circ} 04^{\circ} 08^{\prime}, 00^{\prime \prime}$,

$30^{\circ} 06^{\prime} 00^{\prime \prime}$

$29^{\circ} 58^{\prime} 10^{\prime \prime \prime}$

$29^{\circ} 51^{\prime} 38^{\prime \prime}$

$29^{\circ} 52$ ' $077^{\prime \prime}$,

$29^{\circ} 51^{\prime}, 32^{\prime \prime}$,

$29^{\circ} 45^{\prime} 33^{\prime \prime}$

$29^{\circ} 42$ ' $53^{\prime \prime}$

$99^{\circ} 23^{\prime} 40^{\prime \prime}$

$99^{\circ} 19^{\prime} 23^{\prime \prime}$

$99^{\circ} 16^{\prime} 58^{\prime \prime}$

99.11'42'

$98^{\circ} 53^{\prime} 33^{\prime \prime}$

$98^{\circ} 22^{\prime} 58^{\prime \prime}$

$98^{\circ} 11^{\prime} 55^{\prime \prime}$

$98^{\circ} 10^{\prime} 47^{\prime \prime}$

$98^{\circ} 08^{\prime} 23^{\prime \prime}$

$98^{\circ} 06^{\prime} 35^{\prime \prime}$

$29^{\circ} 43^{\prime} 14^{\prime \prime}, \quad 98^{\circ} 07^{\prime} 23^{\prime \prime}$

$29^{\circ} 42^{\prime} 47^{\prime \prime}, \quad 98^{\circ} 08^{\prime} 14^{\prime \prime}$

$29^{\circ} 42^{\prime} 21^{\prime \prime}, \quad 98^{\circ} 07^{\prime} 20^{\prime \prime}$

$29^{\circ} 41^{\prime} 52^{\prime \prime}, \quad 98^{\circ} 06^{\prime} 23^{\prime \prime}$

$29^{\circ} 40^{\prime} 00^{\prime \prime}, \quad 98^{\circ} 04^{\prime} 14^{\prime \prime}$

$29^{\circ} 52^{\prime} 06^{\prime \prime}, \quad 97^{\circ} 55^{\prime} 38^{\prime \prime}$

$29^{\circ} 59^{\prime} 39^{\prime \prime}, \quad 98^{\circ} 05^{\prime} 19^{\prime \prime}$

$29^{\circ} 58^{\prime} 45^{\prime \prime}$

$29^{\circ} 39^{\prime} 54^{\prime \prime}$

$29^{\circ} 55^{\prime}, 22^{\prime \prime \prime}$,

$29^{\circ} 41^{\prime} 58^{\prime \prime}$

$29^{\circ} 29^{\prime}, 49^{\prime \prime}$,

$29^{\circ} 03^{\prime} 57^{\prime \prime \prime}$,

$28^{\circ} 47^{\prime} 34^{\prime \prime}$,

$97^{\circ} 54^{\circ} 35^{\prime \prime}$

$97^{\circ} 38^{\prime} 59^{\prime \prime}$

$97^{\circ} 40^{\prime} 44^{\prime \prime}$

$97^{\circ} 36^{\prime} 12^{\prime \prime}$

$97^{\circ} 27^{\prime} 17^{\prime \prime}$

$97^{\circ} 26^{\prime} 57^{\prime \prime}$

$97^{\circ} 19^{\prime} 16^{\prime \prime}$

$97^{\circ} 19^{\prime} 16^{\prime \prime}$
$97^{\circ} 00^{\prime} 46^{\prime \prime}$

$28^{\circ} 53^{\prime} 51^{\prime \prime}, \quad 97^{\circ} 21^{\prime} 17^{\prime \prime}$

$28^{\circ} 51^{\prime} 41^{\prime \prime}, \quad 97^{\circ} 13^{\prime} 34^{\prime \prime}$

$97^{\circ} 11^{\prime} 20^{\prime \prime}$

$28^{\circ} 50^{\prime} 21^{\prime \prime}$,

$28^{\circ} 45^{\prime} 04^{\prime \prime}$,

$97^{\circ} 10^{\prime} 47^{\prime \prime}$

$28^{\circ} 45^{\prime} 05^{\prime \prime}$

$97^{\circ} 19^{\prime} 01^{\prime \prime}$

$28^{\circ} 42$ '54",

$97^{\circ} 12$ '42"

$28^{\circ} 43^{\prime} 27^{\prime \prime}, \quad 97^{\circ} 12^{\prime} 07^{\prime \prime}$

$28^{\circ} 43^{\prime} 51^{\prime \prime}, \quad 97^{\circ} 09^{\prime} 53^{\prime \prime}$

$\begin{array}{ll}28^{\circ} 43^{\prime} 54^{\prime \prime}, & 97^{\circ} 09^{\prime} 50^{\prime \prime} \\ 28^{\circ} 43^{\prime} 51^{\prime \prime}, & 97^{\circ} 08^{\prime} 18^{\prime \prime}\end{array}$ $29^{\circ} 41^{\prime} 52^{\prime \prime} \quad 98^{\circ} 08^{\prime} 11^{\prime \prime}$
Austin

Do.

Lo.

Do.

Do. an Antonio

Do.

Do.

Do.

Do.

$\begin{array}{ll}\text { D } & \text { San Angelo } \\ \text { D } & \text { Do. } \\ \text { D } & \text { Do. } \\ \text { D } & \text { Do. } \\ \text { D } & \text { San Antonio } \\ \text { D } & \text { Do. } \\ \text { R } & \text { d/ } \\ \text { DTC } & \text { San Antonio } \\ \text { L } & \text { Do. } \\ \text { D } & \text { Do. }\end{array}$

L Do.

L Do.

Do.

Do.

OpQpPw Do.

$\mathrm{D}$ Do.

D Do.

Do.

DQp Pw Do.

$\mathrm{DQcTc}$ Do.

Do

DQp Do.

$\begin{array}{ll}\text { DBpOpQmQpSpNA } & \text { Do. }\end{array}$

D Do.

Do.

Do.

Do.

Do.

Do.

Do.

Do.

Do.

a/ Quality-of-water records are filed in the District office at Austin, Tex.

d/ Discharge and/or reservoir-content records are furnished by the Corps of Engineers. 
Table 1.--Streamflow, quality, reservoir-content, and partial-record stations maintained by the U.S. Geological Survey in cooperation with State and federal agencies.--Continued

\begin{tabular}{|c|c|c|c|c|}
\hline $\begin{array}{c}\text { Permanent } \\
\text { station } \\
\text { No. }\end{array}$ & Station & $\begin{array}{c}\text { Latitude } \\
\text { and } \\
\text { longitude }\end{array}$ & $\begin{array}{l}\text { Type } \\
\text { of } \\
\text { data }\end{array}$ & $\begin{array}{l}\text { Location } \\
\text { ot } \\
\text { record a/ }\end{array}$ \\
\hline
\end{tabular}

GUADALUPE RIVER BASIN--Continued

Guadalupe River:

San Antonio:

08177700 Olmos Creek at Dresden Drive, San Antonio, TX

08177800

Olmos Reservoir at San Antonio, TX

$08177820 \quad 01 \mathrm{mos}$ Creek at Hildebrandt Ave., San Antonio, TX

08177900

San Antonio River at Navarro Street, San Antonio. $\mathrm{TX}$

08177920

San Antonio River at Dolorosa Street,

San Antonio, TX

08178000 San Antonio River at San Antonio, TX

08178100

08178350

San Pedro Creek at Santa Rosa Street, San Antonio, TX

Alazan Creek:

Martinez Creek at Fredericksburg Road, San Antonio, TX

08178400

08178450

08178500

08178550

08178620

Alazan Creek at West Martin Street, San Antonio, TX

Apache Creek at South Zarzamora Street, San Antonio, TX

San Pedro Creek at Furnish Street, San Antonio, TX

San Antonio River at Ashley Street (Berg's Mil1). San Antonio, TX

Salado Creek:

Lorence Creek at Thousand Oaks Boulevard, San Antonio, TX

Mud Creek:

West Elm Creek at San Antonio, TX

8178640

East E1m Creek at San Antonio, TX

08178650

08178700

08178800

08178880

08179500

08180000

08180700

08180800

08181400

08181480

08181500

08181800

08183500

08183900

08185000

08186000

08186500

08188500

08188600

08188750

Elm Creek Reservoir Site 11 at

San Antonio, TX

Salado Creek (upper station) at San Antonio, TX

Salado Creek (lower station) at San Antonio, TX

Medina River at Bandera, TX

Medina Lake near San Antonio, TX

Medina River:

Diversion Lake:

Medina Canal near Riomedina, TX

Medina River near Macdona, TX

Medina River near Somerset, TX Leon Creek:

Culebra Creek:

Helotes Creek at Helotes, TX

Leon Creek at Interstate Highway 35 at San Antonio, TX

Medina River at San Antonio, TX

San Antonio River near Elmendorf, TX

San Antonio River near Falls City, TX

Cibolo Creek near Boerne, TX

Cibolo Creek at Selma, TX

Cibolo Creek near Falls City, TX

Ecleto Creek near Runge, TX

San Antonio River at Goliad, TX

Guadalupe-Blanco River Authority Calhoun Cana1Flume No. 1 near Long Mott, TX

Guadalupe-Blanco River Authority Calhoun Cana1Flume No. 2 near Long Mott, TX

08188800 Guadalupe River near Tivoli, TX

\section{COPANO CREEK BASIN}

\begin{tabular}{|c|c|c|}
\hline $\begin{array}{l}29^{\circ} 29^{\prime} 56^{\prime \prime}, \\
29^{\circ} 28^{\prime} 28^{\prime \prime}, \\
29^{\circ} 27^{\prime} 56^{\prime \prime}, \\
29^{\circ} 25^{\prime} 50^{\prime \prime},\end{array}$ & $\begin{array}{l}98^{\circ} 30^{\prime} 36^{\prime \prime} \\
98^{\circ} 28^{\prime} 23^{\prime \prime} \\
98^{\circ} 28^{\prime} 01^{\prime \prime} \\
98^{\circ} 29^{\prime} 24^{\prime \prime}\end{array}$ & $\begin{array}{l}\text { HBp 0pPwQmQpQpo San } \\
\text { R } \\
\text { H } \\
\text { F }\end{array}$ \\
\hline $29^{\circ} 25^{\prime} 24^{\prime \prime}$, & $98^{\circ} 29^{\prime} 32^{\prime \prime}$ & G \\
\hline $\begin{array}{l}29^{\circ} 24^{\prime} 34^{\prime \prime}, \\
29^{\circ} 25^{\prime} 51^{\prime \prime},\end{array}$ & $\begin{array}{l}98^{\circ} 29^{\prime} 41^{\prime \prime} \\
98^{\circ} 29^{\prime} 49^{\prime \prime}\end{array}$ & $\begin{array}{l}\text { DBp0p PwQmQpQpo } \\
F\end{array}$ \\
\hline $29^{\circ} 27^{\prime} 22^{\prime \prime}$, & $98^{\circ} 31^{\prime} 04^{\prime \prime}$ & $\mathrm{F}$ \\
\hline $29^{\circ} 25^{\prime} 51^{\prime \prime}$, & $98^{\circ} 30^{\prime} 57^{\prime \prime}$ & $\mathrm{F}$ \\
\hline $29^{\circ} 24^{\prime} 47^{\prime \prime}$ & $98^{\circ} 31^{\prime} 42^{\prime \prime}$ & $\mathrm{F}$ \\
\hline $29^{\circ} 24^{\prime} 22^{\prime \prime}$, & $98^{\circ} 30^{\prime} 38^{\prime \prime}$ & $F$ \\
\hline $29^{\circ} 20^{\prime} 04^{\prime \prime}$, & $98^{\circ} 27^{\prime} 20^{\prime \prime}$ & $F$ \\
\hline $29^{\circ} 35^{\prime} 24^{\prime \prime}$ & $98^{\circ} 27^{\prime} 47^{\prime \prime}$ & $\begin{array}{l}\text { HBpOp PwQa } \\
\text { QmQpQpo }\end{array}$ \\
\hline $29^{\circ} 37^{\prime} 23^{\prime \prime}$, & $98^{\circ} 26^{\prime} 29^{\prime \prime}$ & $\begin{array}{r}\text { HBp OpPwQa } \\
\text { OmUpQpo }\end{array}$ \\
\hline $29^{\circ} 37^{\prime} 04^{\prime \prime}$ & $98^{\circ} 25^{\prime} 41^{\prime \prime}$ & $\begin{array}{r}\text { HBpOpPwQa } \\
\text { QmQpQpo }\end{array}$ \\
\hline $29^{\circ} 36^{\prime} 11^{\prime \prime}$, & $98^{\circ} 25^{\prime} 50^{\prime \prime}$ & RBpOpHwQmQpQpo \\
\hline $\begin{array}{l}29^{\circ} 30^{\prime} 57^{\prime \prime}, \\
29^{\circ} 21^{\prime} 25^{\prime \prime}, \\
29^{\circ} 43^{\prime} 26^{\prime \prime}, \\
29^{\circ} 32^{\prime} 24^{\prime \prime},\end{array}$ & $\begin{array}{l}98^{\circ} 25^{\prime} 51^{\prime \prime} \\
98^{\circ} 24^{\prime} 45^{\prime \prime} \\
99^{\circ} 04^{\prime} 13^{\prime \prime} \\
98^{\circ} 56^{\prime} 01^{\prime \prime}\end{array}$ & $\begin{array}{l}\text { DBpOp PwQmQpQpo } \\
\text { DBpOpPwQmQpQpo } \\
\text { DBpOp PwQmQpQpo } \\
\text { R }\end{array}$ \\
\hline $\begin{array}{l}29^{\circ} 30^{\prime} 19^{\prime \prime}, \\
29^{\circ} 20^{\prime} 05^{\prime \prime}, \\
29^{\circ} 15^{\prime} 45^{\prime \prime},\end{array}$ & $\begin{array}{l}98^{\circ} 54^{\prime} 11^{\prime \prime} \\
98^{\circ} 41^{\prime} 22^{\prime \prime} \\
98^{\circ} 34^{\prime} 56^{\prime \prime}\end{array}$ & $\begin{array}{l}\mathrm{D} \\
\mathrm{D} \\
\mathrm{D}\end{array}$ \\
\hline $\begin{array}{l}29^{\circ} 34^{\prime} 42^{\prime \prime}, \\
29^{\circ} 19^{\prime} 47^{\prime \prime},\end{array}$ & $\begin{array}{l}98^{\circ} 41^{\prime} 29^{\prime \prime} \\
98^{\circ} 35^{\prime} 02^{\prime \prime}\end{array}$ & $\begin{array}{l}\text { DBpOpPwQmQpQpo } \\
\text { Uc OpQmQpoTc }\end{array}$ \\
\hline $\begin{array}{l}29^{\circ} 15^{\prime} 14^{\prime \prime}, \\
29^{\circ} 14^{\prime} 15^{\prime \prime}, \\
28^{\circ} 57^{\prime} 05^{\prime \prime}, \\
29^{\circ} 46^{\prime} 26^{\prime \prime}, \\
29^{\circ} 35^{\prime} 38^{\prime \prime}, \\
29^{\circ} 00^{\prime} 50^{\prime \prime}, \\
28^{\circ} 55^{\prime} 12^{\prime \prime \prime}, \\
28^{\circ} 38^{\prime} 58^{\prime \prime}, \\
28^{\circ} 29^{\prime} 44^{\prime \prime},\end{array}$ & $\begin{array}{l}98^{\circ} 28^{\prime} 20^{\prime \prime} \\
98^{\circ} 21^{\prime} 4^{\prime \prime} \\
98^{\circ} 03^{\prime} 50^{\prime \prime} \\
98^{\circ} 41^{\prime} 50^{\prime \prime} \\
98^{\circ} 18^{\prime} 39^{\prime \prime} \\
97^{\circ} 55^{\prime} 48^{\prime \prime} \\
97^{\circ} 46^{\prime} 19^{\prime \prime} \\
97^{\circ} 23^{\prime} 04^{\prime \prime} \\
96^{\circ} 46^{\prime} 18^{\prime \prime}\end{array}$ & $\begin{array}{l}\text { DOpQmQpQpo } \\
\text { DUpQmQpoQv } \\
\text { D } \\
\text { D } \\
\text { D } \\
\text { DUc OpTc } \\
\text { D } \\
\text { DQBpOpQmQpoSpNA } \\
\text { DPw }\end{array}$ \\
\hline $28^{\circ} 30^{\prime} 09^{\prime \prime}$, & $96^{\circ} 45^{\prime} 40^{\prime \prime}$ & D \\
\hline $28^{\circ} 30^{\prime} 20^{\prime \prime}$, & $96^{\circ} 53^{\prime} 04^{\prime \prime}$ & $\mathrm{GO}_{p} \mathrm{Ps} \mathrm{Pw}_{w} \mathrm{MQpQpo}$ \\
\hline
\end{tabular}

08189200 Copano Creek near Refugio, TX

$28^{\circ} 18^{\prime} 12^{\prime \prime}, \quad 97^{\circ} 06^{\prime} 44^{\prime \prime} \quad$ DOpQmQpQpo

San Antonio

MISSION RIVER BASIN

08189500 Mission River at Refugio, TX

$28^{\circ} 17^{\prime} 30^{\prime \prime}, \quad 97^{\circ} 16^{\prime} 44^{\prime \prime}$ DBpOpQmQpSpNA San Antonio

ARANSAS RIVER BASIN

08189700 Aransas River near Skidmore, TX

$08189800 \quad$ Chiltipin Creek at Sinton, TX

$28^{\circ} 16^{\prime} 56^{\prime \prime}, \quad 97^{\circ} 37^{\prime} 14^{\prime \prime} \quad D$

Do.

\section{NUECES RIVER BAS IN}

08190000 Nueces River at Laguna, TX

08190500 West Nueces River near Brackettville, TX

08192000 Nueces River below Uvalde, TX

08193000 Nueces River near Asherton, TX

08194000 Nueces River at Cotulla, TX

08194200 San Casimiro Creek near Freer, TX

$29^{\circ} 25^{\prime} 42^{\prime \prime}, \quad 99^{\circ} 59^{\prime} 49^{\prime \prime}$ $29^{\circ} 28^{\prime} 21^{\prime \prime}, 100^{\circ} 14^{\prime} 10^{\prime \prime}$ $29^{\circ} 07^{\prime} 25^{\prime \prime}, 99^{\circ} 53^{\prime} 40^{\prime \prime}$ $28^{\circ} 30^{\prime} 01^{\prime \prime}, \quad 99^{\circ} 40^{\prime} 55^{\prime \prime}$ $28^{\circ} 25^{\prime} 34^{\prime \prime}, 99^{\circ} 14^{\prime} 23^{\prime \prime}$ $27^{\circ} 57^{\prime} 53^{\prime \prime}, \quad 98^{\circ} 58^{\prime} 00^{\prime \prime}$

$\begin{array}{ll}\text { DBpUpPwQmQpQpo } & \text { San } \\ \text { D } & \text { Do. } \\ \text { D } & \text { Do. } \\ \text { D } & \text { Do. } \\ \text { D } & \text { Do. } \\ \text { D } & \text { Do. }\end{array}$

a/ Quality-of-water records are filed in the District Office at Austin, Tex. 
Table 1.--Streamflow, quality, reservoir-content, and partial-record stations maintained by the U.S. Geological Survey in cooperation with State and Federal agencies.--Continued

\begin{tabular}{|c|c|c|c|c|}
\hline $\begin{array}{c}\text { Permanent } \\
\text { station } \\
\text { No. }\end{array}$ & Station & $\begin{array}{c}\text { Latitude } \\
\text { and } \\
\text { longitude }\end{array}$ & $\begin{array}{l}\text { Type } \\
\text { of } \\
\text { data }\end{array}$ & $\begin{array}{c}\text { Location } \\
\text { ot } \\
\text { record a/ }\end{array}$ \\
\hline
\end{tabular}

NUECES RIVER BASIN--Continued 08194500
08195000

08196000

08197500

08198000

08198500

08200000

08200700

08201500

08202700

08204000

08205500

08206600

08206700

08206900

08207220

08207300

08208000

08210000

08210400

08210500

08211000

08211500

Nueces River near Tilden, TX

Frio River at Concan, TX

Dry Frio River near Reagan Wells, TX

Frio River below Dry Frio River near Uvalde, TX

Sabinal River near Sabinal, TX

Sabinal River at Sabinal, TX

Hondo Creek near Tarpley, TX

Hondo Creek at King Waterhole near Hondo, TX

Seco Creek at Miller Ranch near Utopia, TX

Seco Creek at Rowe Ranch near D'Hanis, TX

Leona River spring flow near Uvalde, TX (9)

Frio River near Derby, TX

Frio River at Tilden, TX

San Miguel Creek near Tilden, TX Atascosa River:

Rutledge Hollow Creek at 7 th Street, Poteet, TX

Atascosa River at Pleasanton, TX

Atascosa River at Whitsett, TX

Nueces River near Three Rivers, TX

Lagarto Creek near George West, TX

Lake Corpus Christi near Mathis, TX

Nueces River near Mathis, TX

Nueces River at Calallen, TX

08211520 Oso Creek at Corpus Christi, TX
Leona River:

Choke Canyon Reservoir near Three Rivers, TX

$28^{\circ} 18^{\prime} 31^{\prime \prime}$,

$29^{\circ} 30^{\prime} 16^{\prime \prime}$

$29^{\circ} 14^{\prime} 44^{\prime \prime}$

29.29'35",

$29^{\circ} 18^{\prime} 47^{\prime \prime}$,

$29^{\circ} 34^{\prime} 10^{\prime \prime}$

$29^{\circ} 23^{\prime} 26^{\prime \prime}$,

$29^{\circ} 34^{\prime} 23^{\prime \prime}$

$29^{\circ} 21^{\prime} 43^{\prime \prime}$,

29०09'15",

$28^{\circ} 44^{\prime} 11^{\prime \prime}$

$28^{\circ} 28^{\prime} 02^{\prime \prime}$

$28^{\circ} 35^{\prime} 14^{\prime \prime}$

$28^{\circ} 29^{\prime} 01^{\prime \prime}$,

$29^{\circ} 02^{\prime} 07^{\prime \prime}$

$28^{\circ} 57^{\prime} 44^{\prime \prime}$,

$28^{\circ} 37^{\prime} 18^{\prime \prime}$,
$28^{\circ} 26^{\prime} 10^{\prime \prime}$,

$28^{\circ} 03^{\prime} 34^{\prime \prime}$

$28^{\circ} 02$ ' $17^{\prime \prime}$,

$28^{\circ} 02 ' 17^{\prime \prime}$,
$27^{\circ} 52$ ' $40^{\prime \prime}$,

$98^{\circ} 33^{\prime} 25^{\prime \prime}$ $99^{\circ} 42^{\prime} 16^{\prime \prime}$ $99^{\circ} 46^{\prime} 52^{\prime \prime}$ $99^{\circ} 40^{\prime} 27^{\prime \prime}$ $99^{\circ} 29^{\prime} 49^{\prime \prime}$ $99^{\circ} 28^{\prime} 46^{\prime \prime}$ $99^{\circ} 14^{\prime} 47^{\prime \prime}$ $99^{\circ} 09^{\prime} 04^{\prime \prime}$ $9^{\circ} 24^{\prime} 10^{\prime \prime}$ $99^{\circ} 17^{\prime} 05^{\prime \prime}$

D DBpOpPwQmQpQpo DBpOpPwQmQpQpo DBpOp PwQmQpQpo DBpOp PwQmQpQpo DBpup YwQmQpQpo

$99^{\circ} 44^{\prime} 35^{\prime \prime}$ $99^{\circ} 08^{\prime} 40^{\prime \prime}$ $98^{\circ} 32^{\prime} 50^{\prime \prime}$ $98^{\circ} 32^{\prime} 44^{\prime \prime}$ $98^{\circ} 14^{\prime} 44^{\prime \prime}$

$98^{\circ} 34^{\prime} 18^{\prime \prime}$ $98^{\circ} 28^{\prime} 51^{\prime \prime}$ $98^{\circ} 17^{\prime} 02^{\prime \prime}$ $98^{\circ} 11^{\prime} 06^{\prime \prime}$

$98^{\circ} 05^{\prime} 48^{\prime \prime}$ $97^{\circ} 52^{\prime} 15^{\prime \prime}$ $97^{\circ} 51^{\prime} 36^{\prime \prime}$ $97^{\circ} 37^{\prime} 35^{\prime \prime}$

OSU CREEK BASIN

$27^{\circ} 42^{\prime} 40^{\prime \prime}, \quad 97^{\circ} 30^{\prime} 06^{\prime \prime}$ DOPQmQpQpo

SAN FERNANDO CREEK BASIN

08211800 San Diego Creek at Alice, TX (head of San Fernando Creek)

$27^{\circ} 45^{\prime} 59^{\prime \prime}, \quad 98^{\circ} 04^{\prime} 31^{\prime \prime}$

$27^{\circ} 47^{\prime} 25^{\prime \prime}, \quad 98^{\circ} 03^{\prime} 39^{\prime \prime} \quad \mathrm{R}$

aly

08211850

08211900

08212300

San Fernando Creek at Alice, TX

Tranquitas Creek at Kingsville, TX

$27^{\circ} 31^{\prime} 33^{\prime \prime}, 97^{\circ} 52^{\prime} 02^{\prime \prime}$

\section{RIO GRANDE BASIN}

08364000 Rio Grande near El Paso, TX

08370500 Rio Grande below Old Fort Quitman, TX

08377200

08407500

08408500

08410000

08412500

08414500

08415000

08418000

08425500

08427000

08427500

08431700

08436500

08437500

08437600

08437700

08446500

08447410

08449400

08450900

08456300

08459000

08461200

08461300

08464700

08466300

08467800

08469200

08475000
Rio Grande at Foster Ranch near Langtry, TX

Pecos River at Red Bluff, NM

Delaware River near Red Bluff, NM

Red Bluff Reservoir near Orla, TX

Pecos River near Orla, TX

Reeves County W.I.D. No. 2 canal near Mentone, TX

Ward County W.I.D. No. 3 canal near Barstow, TX

Ward County Irrigation District No. 1 canal near Barstow, TX

Toyah Creek:

Phantom Lake Spring near Toyahvale, TX (9)

Giffin Springs at Toyahvale, TX (2)

San Solomon Spring at Toyahvale, TX (9)

Limpia Creek above Fort Davis, TX

(9)

Pecos County W.I.D. No. 2 upper diversion canal near Grandfalis, TX

Pecos County W.I.D. No. 2 canal near Imperial, TX

Pecos County W.I.D. No. 3 canal near Imperial, TX

Ward County W.I.D. No. 2 canal near Grandfalls, TX

Pecos River near Girvin, TX

Pecos River near Langtry, TX

Devils River at Pafford Crossing near Comstock, TX

Rio Grande below Amistad Dam near Del Rio, TX

Las Moras Creek:

Las Moras Springs at Brackettville, TX

Rio Grande at Laredo, TX

International Falcon Reservoir near Falcon Heights, TX

Rio Grande below Falcon Dam, TX

Rio Grande at Fort Ringgold, Rio Grande City, TX

Rio Grande near Los Ebanos, TX

Morillo Drain, Mex.

Rio Grande below Anzalduas Dam, TX

Rio Grande at Brownsville, TX

$31^{\circ} 48^{\prime} 10^{\prime \prime}, 106^{\circ} 32^{\prime} 25^{\prime \prime}$

$29^{\circ} 46^{\prime} 50^{\prime \prime}, 101^{\circ} 45^{\prime} 20^{\prime \prime}$

$32^{\circ} 04^{\prime} 30^{\prime \prime}, 104^{\circ} 02^{\prime}, 21^{\prime \prime}$

$32^{\circ} 01^{\prime} 23^{\prime \prime}, 104^{\circ} 03^{\prime} 15^{\prime \prime}$

$31^{\circ} 54^{\prime} 04^{\prime \prime}, 103^{\circ} 54^{\prime} 35^{\prime \prime}$

$31^{\circ} 52^{\prime} 21^{\prime \prime}, 103^{\circ} 49^{\prime} 52^{\prime \prime}$

$31^{\circ} 37^{\prime} 57^{\prime \prime}, 103^{\circ} 34^{\prime} 30^{\prime \prime}$

$31^{\circ} 34^{\prime} 28^{\prime \prime}, 103^{\circ} 30^{\prime} 04^{\prime \prime}$

$31^{\circ} 32$ ' $26^{\prime \prime}, 103^{\circ} 29^{\prime} 42^{\prime \prime}$

$103^{\circ} 29^{\prime} 42^{\prime \prime} \mathrm{D}$

$30^{\circ} 56^{\prime} 01^{\prime \prime}, 103^{\circ} 50^{\prime} 43^{\prime \prime}$

$30^{\circ} 56^{\prime} 51^{\prime \prime \prime}, 103^{\circ} 47^{\prime} 19^{\prime \prime}$

$30^{\circ} 56^{\prime} 34^{\prime \prime}, 103^{\circ} 47^{\prime} 16^{\prime \prime}$

$30^{\circ} 36^{\prime} 48^{\prime \prime}, 104^{\circ} 00^{\prime} 04^{\prime \prime}$
$31^{\circ} 18^{\prime} 43^{\prime \prime}, 102^{\circ} 55^{\prime} 10^{\prime \prime}$

$31^{\circ} 16^{\prime} 38^{\prime \prime}, 102^{\circ} 43^{\prime} 54^{\prime \prime}$

$31^{\circ} 06^{\prime} 47^{\prime \prime}, 102^{\circ} 25^{\prime} 02^{\prime \prime}$

$29^{\circ} 48^{\prime} 10^{\prime \prime}, 101^{\circ} 26^{\prime} 45^{\prime \prime}$

$29^{\circ} 40^{\prime} 35^{\prime \prime}, 101^{\circ} 00^{\prime} 00^{\prime \prime}$

$29^{\circ} 25^{\prime} 00^{\prime \prime}, 101^{\circ} 02^{\prime} 00^{\prime \prime}$

$29^{\circ} 18^{\prime} 33^{\prime \prime}, 100^{\circ} 25^{\prime} 13^{\prime \prime}$

$27^{\circ} 29^{\prime} 45^{\prime \prime}, \quad 99^{\circ} 29^{\prime} 25^{\prime \prime}$

$26^{\circ} 33^{\prime} 25^{\prime \prime}, 99^{\circ} 10^{\prime} 05^{\prime \prime}$

$26^{\circ} 33^{\prime} 25^{\prime \prime}, \quad 99^{\circ} 10^{\prime} 05^{\prime \prime}$

$26^{\circ} 22^{\prime} 05^{\prime \prime}, 98^{\circ} 48^{\prime} 20^{\prime \prime}$

$26^{\circ} 14^{\prime} 15^{\prime \prime}, \quad 98^{\circ} 33^{\prime} 50^{\prime \prime}$

$26^{\circ} 08^{\prime} 00^{\prime \prime}, \quad 98^{\circ} 20^{\prime} 05^{\prime \prime}$

$25^{\circ} 52$ '35"', $97^{\circ} 27^{\prime} 15^{\prime \prime}$

BpOpQmQpSpNA BPUPQmQpSPNA BpOPUmUPSPNA D

$\mathrm{DQ}$

D

D

L
L
DBPOpQmQpSp

BpOpQmQpSp$$
\text { D }
$$

\section{$D$}

QpBpOpUmSpNA

QpBPUp(mSPNA

QP

L

QBPUPQ⿴囗十⺝SPNA

Qp

Qp

QP

BpOpQmUpSpNA $31^{\circ} 26^{\prime} 51^{\prime \prime}, 102^{\circ} 44^{\prime} 26^{\prime \prime}$
San Antonio

San Antonio

Do .

Do.

Do.

Do.

Do.

Do.

uo.

Do.

Do.

Do.

Do.

Do.

Do.

Lo.

Do.

Do.

Do.

Do.

San Antonio

Do.

Do.

Do.

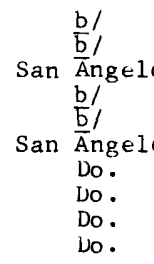

Do.

Do.

Do.

Do.

Do.

Lo.

San Angelo

Do.

I.B. \& W.C.

Do.

Do.

Do.

Do.

Do.

עo.

Do.

a/ Quality-of-water records are filed in the District Office at Austin, Tex.

b/ Records are furnished by adjacent USGS District Offices. 